\title{
Spontaneous and induced dynamic correlations in glass formers. II. Model calculations and comparison to numerical simulations
}

\author{
L. Berthier \\ Laboratoire des Colloüdes, Verres et Nanomatériaux, UMR 5587, Université Montpellier II and CNRS, \\ 34095 Montpellier, France \\ G. Biroli \\ Service de Physique Théorique Orme des Merisiers, CEA Saclay, 91191 Gif sur Yvette Cedex, France \\ J.-P. Bouchaud \\ Service de Physique de l'Etat Condense Orme des Merisiers, CEA Saclay, 91191 Gif sur Yvette Cedex, \\ France and Science and Finance, Capital Fund Management, 6-8 Boulevard Haussmann, 75009 Paris, \\ France \\ W. Kob \\ Laboratoire des Colloïdes, Verres et Nanomatériaux, UMR 5587, Université Montpellier II and CNRS, \\ 34095 Montpellier, France \\ K. Miyazaki and D. R. Reichman \\ Department of Chemistry, Columbia University, New York, New York 10027
}

(Received 29 September 2006; accepted 12 March 2007; published online 8 May 2007)

\begin{abstract}
We study in detail the predictions of various theoretical approaches, in particular, mode-coupling theory (MCT) and kinetically constrained models (KCMs), concerning the time, temperature, and wave vector dependence of multipoint correlation functions that quantify the strength of both induced and spontaneous dynamical fluctuations. We also discuss the precise predictions of MCT concerning the statistical ensemble and microscopic dynamics dependence of these multipoint correlation functions. These predictions are compared to simulations of model fragile and strong glass-forming liquids. Overall, MCT fares quite well in the fragile case, in particular, explaining the observed crucial role of the statistical ensemble and microscopic dynamics, while MCT predictions do not seem to hold in the strong case. KCMs provide a simplified framework for understanding how these multipoint correlation functions may encode dynamic correlations in glassy materials. However, our analysis highlights important unresolved questions concerning the application of KCMs to supercooled liquids. (C) 2007 American Institute of Physics. [DOI: 10.1063/1.2721555]
\end{abstract}

\section{INTRODUCTION}

Dynamic heterogeneity is a well established feature of the behavior of a diverse class of systems close to their glass transition temperatures. ${ }^{1-6}$ Given the relatively recent realization of the importance of dynamic heterogeneity, it is not surprising that the systematic characterization of such spatiotemporal behavior, and the length scales associated with it, is far from complete. Much recent effort has been expended to devise correlation functions that effectively and quantitatively probe dynamic heterogeneity. ${ }^{7-14}$ The theoretical understanding of the behavior of such correlation functions is still in its infancy. This is to be contrasted with our relatively mature understanding of bulk structure and dynamics in supercooled liquids as measured by simple, low order correlation functions (such as intermediate scattering functions) that can only indirectly hint at dynamic heterogeneity. ${ }^{1,15}$

In the first paper of this series, denoted in the following as $I,{ }^{16}$ we set out to provide a general understanding of the behavior of a particular class of multipoint correlation functions that encode information concerning the growing dynamical length scale in supercooled liquids. To set the stage for the present work we briefly recall some definitions and results obtained in I. Let $f(\mathbf{r}, t)=o(\mathbf{r}, t) o(\mathbf{r}, 0)$ be the instantaneous value of a local two-time correlator at position $\mathbf{r}$ and time $t$, and $[f(t)]_{\mathbf{r}}=V^{-1} \int d^{d} \mathbf{r} f(\mathbf{r}, t)$ its spatial average over a large but finite volume $V$. The thermal average $\left\langle[f(t)]_{\mathrm{r}}\right\rangle$ is a standard two-time correlator, such as the intermediate scattering function when the observable $o(\mathbf{r}, t)$ is the excess density $\rho(\mathbf{r}, t)-\rho_{0}$. A previously defined multipoint susceptibility is the following four-point dynamic susceptibility;

$$
\chi_{4}(t)=N\left\langle[\delta f(t)]_{\mathbf{r}}^{2}\right\rangle=\rho \int d^{d} \mathbf{r}\langle\delta f(\mathbf{r}, t) \delta f(\mathbf{0}, t)\rangle,
$$

where we introduced the notation $\delta X \equiv X-\langle X\rangle$ for the fluctuations of the observable $X$. From Eq. (1), we see that $\chi_{4}(t)$ quantifies the strength of spontaneous fluctuations of the dynamical behavior in supercooled liquids by their variance. As shown by the last term in Eq. (1) fluctuations become larger if this dynamic heterogeneity becomes increasingly spatially correlated. Since $\chi_{4}(t)$ is the volume integral of the fourpoint correlator $S_{4}(\mathbf{r}, t)=\langle\delta f(\mathbf{r}, t) \delta f(\mathbf{0}, t)\rangle$ [or, alternatively, in Fourier space, $\left.\chi_{4}(t)=\lim _{\mathbf{q} \rightarrow 0} S_{4}(\mathbf{q}, t)\right]$, it is directly related to the number of correlated particles, $\chi_{4}(t) \sim(\xi / a)^{d_{f}}$, where $\xi$ is the dynamic correlation length, $a$ a molecular length scale, 
and $d_{f}$ is related to the possibly fractal geometry of the dynamic heterogeneity. The direct link between $\chi_{4}(t)$ and the length scale of dynamic heterogeneity $\xi$ explains the intensity of the present experimental effort dedicated to its measurement. ${ }^{17-20}$

Recognizing that spontaneous fluctuations are in general hard to access experimentally, we have suggested ${ }^{16,19}$ to measure instead the response of the averaged two-time dynamical correlators to an infinitesimal perturbing field,

$$
\chi_{x}(t)=\frac{\partial\left\langle[f(t)]_{\mathbf{r}}\right\rangle}{\partial x}
$$

In particular, we have dedicated much effort to the cases where $x$ is either the temperature, $x=T$, or the density, $x=\rho$, focusing therefore on $\chi_{T}(t)$ and $\chi_{\rho}(t)$. In Ref. 19 it was argued that Eq. (2) defines an experimentally accessible multipoint dynamic susceptibility which is a relevant alternative to $\chi_{4}(t)$. There are two important arguments to support this claim, which we only summarize for $\chi_{T}(t)$, but they directly carry over to $\chi_{\rho}(t)$. The first one is that for a classical fluid evolving via Newton's equations at constant number of particles $N$, pressure $P$, and enthalpy $H$, the following fluctuation-dissipation theorem holds:

$$
k_{B} T^{2} \chi_{T}(t)=V\left\langle[\delta f(t)]_{\mathbf{r}}[\delta h(t)]_{\mathbf{r}}\right\rangle=\int d^{d} \mathbf{r}\langle\delta f(\mathbf{r}, t) \delta h(\mathbf{0}, 0)\rangle,
$$

where $[h(t)]_{\mathbf{r}}=V^{-1} \int d^{d} \mathbf{r} h(\mathbf{r}, t)$ is the instantaneous value of the enthalpy density, and $k_{B}$ the Boltzmann constant. The similarity between Eqs. (1) and (3) is striking. The new susceptibility $\chi_{T}(t)$ quantifies the strength of correlations between dynamic fluctuations and energy fluctuations. As shown by the last term in Eq. (3) $\chi_{T}(t)$ becomes larger if dynamical and energy fluctuations become increasingly spatially correlated. Since $\chi_{T}(t)$ is proportional to the volume integral of the three-point correlator $S_{T}(\mathbf{r}, t)$ $=\langle\delta f(\mathbf{r}, t) \delta h(\mathbf{0}, 0)\rangle$ [or, alternatively, $\chi_{T}(t)=\lim _{\mathbf{q} \rightarrow 0} S_{T}(\mathbf{q}, t)$, it is also directly related to a correlation volume, which makes it an equally appealing quantity. A second argument establishing the relevance of $\chi_{T}(t)$ is the fact that $\chi_{T}(t)$ and $\chi_{4}(t)$ can be related by the following inequality:

$$
\chi_{4}(t) \geqslant \frac{1}{c_{P}} T^{2} \chi_{T}^{2}(t),
$$

where $c_{P}=V\left\langle[\delta h(t)]_{\mathbf{r}}^{2}\right\rangle /\left(\rho T^{2}\right)$ is the constant pressure specific heat expressed in units of $k_{B}$. The result (4) can be understood by formal consideration about statistical ensembles (see I) or more simply by noting that the relation (4) stems from the fact that the (squared) cross correlation between two observables [encoded in $\chi_{T}(t)$ ] cannot be larger than the product of their variances [encoded in $\chi_{4}(t)$ and $c_{P}$ ].

In I we focused on the thermodynamic ensemble dependence and the dependence on the microscopic dynamics. Using general theoretical arguments, we gave qualitative and quantitative guidelines for these dependences. The ensemble variability of global multipoint indicators of dynamical heterogeneity (corresponding to fluctuations of intensive dynamical correlators) is not surprising, given what is already understood about the ensemble dependence of simpler susceptibilities near standard critical points. ${ }^{21,22}$ Importantly, this ensemble dependence allows for the derivation of the rigorous bound (4) on $\chi_{4}(t)$ that is potentially useful for providing a simple experimental estimate of the length scale associated with dynamical heterogeneity near $T_{g}$. That this bound becomes a good approximation for $\chi_{4}(t)$ above $T_{g}$ was checked in simulations of both strong and fragile glassforming liquids in I. The predicted dependence on the underlying nature of the dynamics is perhaps more surprising, especially in light of the fact that simulations of simple dynamical correlation functions show no such nontrivial dependence. ${ }^{23-25}$ Again, in I we have confirmed this striking prediction by atomistic simulations.

Having outlined some generic properties of a class of multipoint indicators of dynamical heterogeneity, and confirmed these basic predictions in I, we now turn to the information contained in specific theories of glassy dynamics. In particular, we address in this paper various properties of these susceptibilities from the standpoint of simple meanfield spin-glass models ${ }^{26}$ the mode-coupling theory (MCT) of supercooled liquids, ${ }^{27}$ and kinetically constrained models (KCMs). ${ }^{28}$ Our choice of theoretical models is natural: to our knowledge, only MCT and KCMs offer a detailed theoretical description of dynamic heterogeneity in supercooled liquids. We aim to confront these theories with the general theoretical properties outlined in I, as well as with simulations of atomistic glass-forming systems. The outcome of this exercise will be a greater understanding of the successes and failures of these theories, which will lead us to formulate a number of questions related to the comparison of these models with the expectations outlined in I.

This paper is organized as follows: In Sec. II, we present the results predicted by MCT. This includes general scaling behavior, as well as the dynamics and ensemble dependence as derived via a field-theoretical approach to MCT. Within this approach we can show, in particular, that strong ensemble and dynamics dependence of dynamic fluctuations arises, while no such dependence is expected for averaged quantities. $^{29}$ This section discusses the wavelength dependence of $\chi_{4}(t)$ for liquid state MCT, while the relationship between $\chi_{4}(t)$ and $\chi_{T}(t)$ within $p$-spin models for which MCT is exact is performed in the Appendixes. In Sec. III we turn to the ensemble and dynamics dependence of $\chi_{4}(t)$ and $\chi_{T}(t)$ in KCMs. Here, we discuss different models with varying degrees of cooperativity. Interesting unresolved questions, concerning the relevance of KCMs to model molecular glasses, are outlined in this section. In Sec. IV, the predictions of these various models are compared to atomistic simulations. In Sec. V, we conclude and we detail the successes and failure of the theoretical models in light of the comparison with simulations and give a summary of these comparisons in Table I.

\section{MODE-COUPLING THEORY OF DYNAMICAL FLUCTUATIONS}

\section{A. MCT and dynamic fluctuations}

Because it starts from a microscopic description of supercooled liquids and ends up with a complete description of 
its dynamics, MCT is a powerful tool for the interpretation and prediction of the qualitative and quantitative behaviors of slow dynamics in glass-forming liquids and colloids, at least not too close to the glass transition. ${ }^{27}$ The MCT transition is usually described as a small scale phenomenon, the self-consistent blocking of the particles in their local cages. ${ }^{27}$ This is surprising since on general grounds a diverging relaxation time is expected to arise from processes involving an infinite number of particles (leaving aside the case of quenched obstacles). ${ }^{30}$ Actually, the cage mechanism requires some kind of correlation in space: in order to be blocked by one's neighbors, the neighbors themselves must be blocked by their neighbors and so on until a certain scale that, intuitively, sets the relaxation time scale of the system. The fact that within MCT "cages" are correlated objects ${ }^{31}$ will, in fact, become clear below.

This "local cage" point of view was challenged in the context of mean-field disordered systems by Franz and Paris ${ }^{11}$ see also Refs. 32 and 33 for early results. For these models the dynamical equations for correlators are formally equivalent to the schematic version of the MCT equations. Franz and Parisi ${ }^{11}$ argued that a dynamical susceptibility similar to $\chi_{4}(t)$ in these models has a diverging peak at the dynamical mode-coupling transition. The Franz-Parisi susceptibility is further discussed in the Appendixes. Although a length scale cannot be defined in mean-field models, a diverging susceptibility is the usual mean-field symptom for a diverging length scale in finite dimensions. More recently, two of the authors (BB), ${ }^{34}$ using a field-theoretical approach to MCT, clearly showed the existence of a diverging length within MCT and analyzed the critical properties of dynamical fluctuations. In that work the role of conserved quantities, emphasized in I, was overlooked. As we show in the following, BB's results for $\chi_{4}(t)$ are correct either for dynamics without any conserved variables (as is the case for disordered $p$-spin systems with Langevin dynamics) or in ensembles where all conserved variables are fixed, i.e., $N V E$ for Newtonian dynamics and NVT for Brownian or Monte Carlo (MC) dynamics.

When there are conserved variables the four-point correlation function $S_{4}(\mathbf{q}, t)$ can be decomposed into two terms, in agreement with the general considerations of I. These two terms reflect different physical contributions for $\mathbf{q}=0$ : one is the contribution in the ensemble where all conserved variables are strictly fixed, and the second arises from the fluctuations of dynamically conserved variables that feed back into the dynamical correlations. The second term (for $q=0$ ) is therefore absent in an ensemble where these variables are fixed. This latter term is the one that yields a lower bound for $\lim _{\mathbf{q} \rightarrow 0} S_{4}(\mathbf{q}, t)$, as expressed in Eq. (4). The bound involves the derivative $\chi_{x}(t)$ defined in Eq. (2), where $x$ is a conserved variable. For example $x=\rho$ for hard spheres, where the density is a conserved quantity both for Brownian and Newtonian dynamics, or $x=H$ (or $E$ ), the enthalpy (or the energy), in cases where temperature is the relevant control parameter. One can of course also focus on dynamical responses with respect to thermodynamic control parameters such as the pressure or the temperature. One formulation is related to the other via a trivial thermodynamic change of variables and the chain rule. In the following, for simplicity, we will always focus on the derivative with respect to conserved degrees of freedom.

In the next subsections we shall uncover the critical properties of the dynamical fluctuations and dynamical responses discussed above and obtain and analyze quantitative predictions for dynamical responses within MCT. We numerically confirm these results within the $p$-spin model in the Appendixes.

\section{B. Dynamic scaling and critical behavior}

In the following, using the field-theoretical framework developed in I, we obtain the critical behavior of dynamical fluctuations close to the MCT transition. We focus, in particular, on $\chi_{4}(t), S_{4}(\mathbf{q}, t)$, and $\chi_{x}(t)$.

\section{Ladder diagrams within MCT}

Different derivations of MCT follow a common strategy: write down exact or phenomenological stochastic equations for the evolution of the slow conserved degrees of freedom and then use a self-consistent one-loop approximation to close the equations. For instance, in the case of Brownian dynamics the only conserved quantity is the density, and the so-called Dean-Kawasaki equation ${ }^{35,36}$ has been analyzed (see Refs. 37-39 for a discussion of the different field theories). Field theories are obtained through the Martin-SiggiaRose-De Dominicis-Janssen method, where one first introduces response fields enforcing the correct time evolution and then averages over the thermal noise. ${ }^{40}$

The direct derivation of MCT equations starting from field theory is difficult and different approaches have been pursued. ${ }^{38}$ It is still unclear how to obtain in a consistent way the standard MCT equations derived by the Mori-Zwanzig formalism. ${ }^{27,39,41}$ Indeed, if time-reversal symmetry is preserved, one-loop self-consistent equations are not the standard MCT equations but have similar qualitative properties. ${ }^{37}$ They lead, in particular, to the same critical behavior of the correlators. This issue is not relevant here because we focus on qualitative properties of dynamic fluctuations which depend only on the critical properties of the MCT transition.

The starting point for describing dynamic fluctuations within field theory is the Legendre functional ${ }^{40,42,43}$ $\Gamma\left(\Psi_{a}, G_{a, b}\right)$ (here and in the following we use the notations introduced in I):

$$
\begin{aligned}
\Gamma\left(\Psi_{a}, G_{a, b}\right)= & -\frac{1}{2} \operatorname{Tr} \log G+\frac{1}{2} \operatorname{Tr} G_{0}^{-1}[G+\Psi \Psi] \\
& -\Phi_{2 \mathrm{PI}}(\Psi, G),
\end{aligned}
$$

where $\Phi_{2 \mathrm{PI}}\left(\Psi_{a}, G_{a, b}\right)$ is the sum of all two-particle irreducible Feynman diagrams (namely, those that cannot be decomposed in two disjoint pieces by cutting two lines) constructed with the vertices of the theory, using the full propagator $G$ as the lines and $\Psi$ as the sources $\left(G_{0}\right.$ is the bare propagator). ${ }^{40,42}$ The first derivative of $\Gamma\left(\Psi_{a}, G_{a, b}\right)$ leads to the self-consistent equations for the order parameters themselves (including $G$ 's), whereas the second derivatives lead to the equation for the fluctuations of the order parameters. 


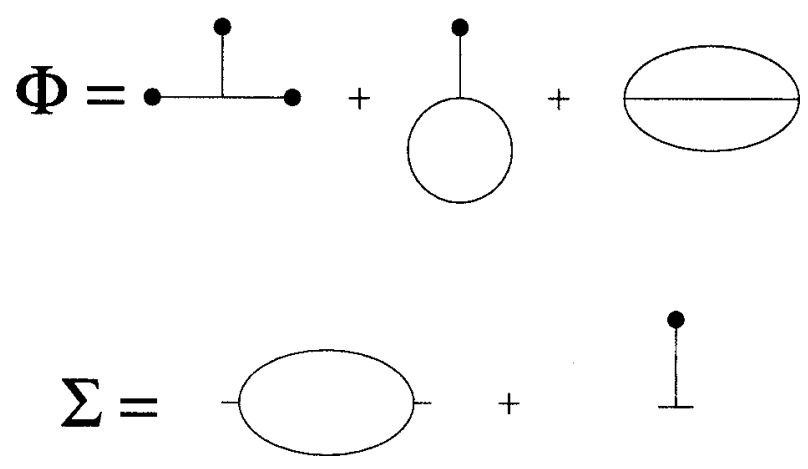

FIG. 1. Three diagrams approximating $\Phi_{2 \mathrm{PI}}\left(\Psi_{a}, G_{a, b}\right)$ within the MCT approximation. The resulting expression of the self-energy is also shown. Lines are full propagators, and dots are conserved variables.

All field-theoretic derivations of MCT consist of oneloop self-consistent equations for the dynamical structure factor. At the level of the functional this corresponds to an approximation of $\Phi_{2 \mathrm{PI}}\left(\Psi_{a}, G_{a, b}\right)$ in which only the first three diagrams of Fig. 1 are considered. They are constructed from a three-leg vertex that is present in all field theories of dense liquids. The black dots represent the $\delta \Psi$ attached as sources and the lines the full propagators of the theory. The corresponding expression of the self-energy $\Sigma=\delta \Phi / \delta G$ is also shown. Note that the second diagram is not present in the usual expression for the self-energy used in the MCT equations because the solution of the self-consistent equation for $\Psi$ leads to $\delta \Psi=0$. As discussed in $\mathrm{I}$, the reason is that the average value of the response fields is zero and the bare values of the physical slow fields are not corrected at any order of the self-consistent expansion because they correspond to conserved variables, whose average value is not fixed by the dynamics but through the initial conditions.

However, when the matrix of second derivatives of $\Gamma$ is considered, it is important to keep the second self-energy diagram because it gives a contribution that cannot be neglected. The matrix of second derivatives reads

$$
\begin{aligned}
& \frac{\delta^{2} \Gamma}{\delta G_{1,2} \delta G_{3,4}}=\left[G_{1,3}^{-1} G_{2,4}^{-1}-\frac{\delta^{2} \Phi_{2 \mathrm{PI}}}{\delta G_{1,2} \delta G_{3,4}}\right], \\
& \frac{\delta^{2} \Gamma}{\delta \Psi_{1} \delta G_{2,3}}=-\frac{\delta^{2} \Phi_{2 \mathrm{PI}}}{\delta \Psi_{1} \delta G_{2,3}}, \\
& \frac{\delta^{2} \Gamma}{\delta \Psi_{1} \delta \Psi_{2}}=\left(G_{0}^{-1}\right)_{1,2} .
\end{aligned}
$$

As in I, we denote these operators, respectively, as $A, B$, and $C$. The diagrammatic expressions for the second derivatives of $\Phi_{2 \text { PI }}$ are shown in Fig. 2. Note that we show only the contributions that are nonzero when evaluated for the average quantities (in particular, for $\delta \Psi=0$ ).

All dynamic fluctuations can then be expressed in terms of $A^{-1}, B, C$ (see I). In particular, the four-point fluctuations

$$
\begin{aligned}
& \left\langle\left\{\tilde{\psi}_{a}(\mathbf{x}, t) \tilde{\psi}_{b}\left(\mathbf{x}^{\prime}, t^{\prime}\right)-\Psi_{a}(\mathbf{x}, t) \Psi_{b}\left(\mathbf{x}^{\prime}, t^{\prime}\right)\right\}\right. \\
& \left.\quad \times\left\{\widetilde{\psi}_{c}(\mathbf{y}, s) \widetilde{\psi}_{d}\left(\mathbf{y}^{\prime}, s^{\prime}\right)-\Psi_{c}(\mathbf{y}, s) \Psi_{d}\left(\mathbf{y}^{\prime}, s^{\prime}\right)\right\}\right\rangle_{c}
\end{aligned}
$$

are given by

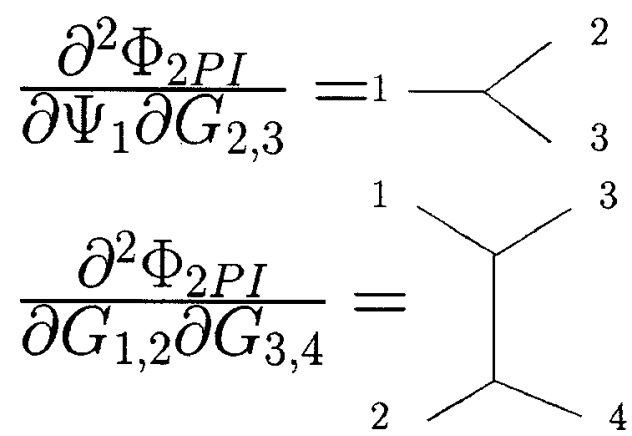

FIG. 2. Diagrammatic expression of $\delta^{2} \Phi_{2 \mathrm{PI}} / \delta \Psi_{1} \delta G_{2,3}$ and $\delta^{2} \Phi_{2 \mathrm{PI}} / \delta G_{1,2} \delta G_{3,4}$ within MCT.

$$
A^{-1}+\left(A^{-1} B\right)\left\{C-B^{\dagger} A^{-1} B\right\}^{-1}\left(A^{-1} B\right)^{\dagger}
$$

evaluated at the matrix element $\left[a, b, \mathbf{x}, \mathbf{x}^{\prime}, t, t^{\prime}\right.$; $\left.c, d, \mathbf{y}, \mathbf{y}^{\prime}, s, s^{\prime}\right]$. The explicit expression for $A^{-1}, B, C$ makes it clear that within MCT the critical properties of dynamical fluctuations come only from $A^{-1}$. Indeed, $C$ is just the inverse of the bare propagator, whereas $B$ is the bare vertex. These quantities have no critical behavior at the MCT transition. Instead, using the general results of I and the MCT expression of $\delta^{2} \Phi_{2 \mathrm{PI}} / \delta G \delta G$, one finds that $A^{-1}$ corresponds to the sum of $n$-ladder diagrams shown in Fig. 3. As shown in Ref. 34 , the resummation of these diagrams indeed leads to a critical contribution at the MCT transition.

In particular, they give a contribution to the four-point function $\left\langle\delta \rho_{-k_{3}}(t) \delta \rho_{k_{3}+q}(0) \delta \rho_{-k_{4}}(t) \delta \rho_{k_{4}-q}(0)\right\rangle$ that scales as $^{31,34}$

$$
\begin{aligned}
& \frac{1}{\sqrt{\epsilon}+q^{2}} g_{\beta}\left(\frac{q^{2}}{\sqrt{\epsilon}}, \frac{t}{\tau_{\beta}}\right), \quad t \propto \tau_{\beta}, \\
& \frac{1}{\sqrt{\epsilon}\left[\sqrt{\epsilon}+q^{2}\right]} g_{\alpha}\left(\frac{t}{\tau_{\alpha}}\right), \quad t \propto \tau_{\alpha} .
\end{aligned}
$$

Note that here and in the following we use the standard MCT notation. ${ }^{27,41}$ In particular, $\epsilon=\left|x_{c}-x\right| / x_{c}$ is the reduced distance from the critical point at $x=x_{c}$. The function $g_{\beta}\left(q^{2} / \sqrt{\epsilon}, t / \tau_{\beta}\right)$ behaves as $\left(t / \tau_{\beta}\right)^{a}$ and $\left(t / \tau_{\beta}\right)^{b}$ for small and large values of $\left(t / \tau_{\beta}\right)$, respectively. Furthermore the function

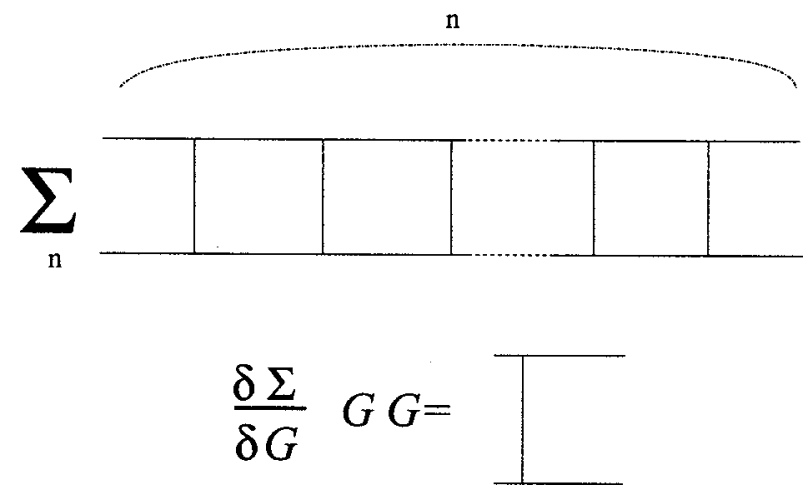

FIG. 3. Expression for $A^{-1}$ within MCT. It consists in a sum of $n$ ladders constructed from the elementary block $(\delta \Sigma / \delta G) G G$ shown in the second line, see Eq. (6). 


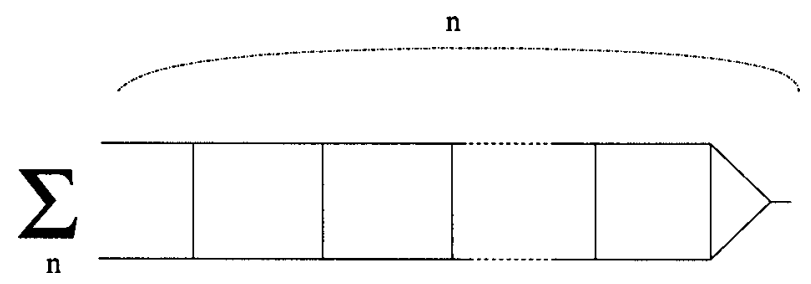

FIG. 4. The dynamical response obtained from Eq. (10) by noting that the inversion of $A=\partial_{G G}^{2} \Gamma$ involves resumming ladders that close to $\partial_{G \Psi}^{2} \Gamma$.

$g_{\alpha}\left(t / \tau_{\alpha}\right)$ behaves as $\left(t / \tau_{\alpha}\right)^{b}$ for small values of $\left(t / \tau_{\alpha}\right)$, see also Eqs. (16) and (17) below.

Below we show that the critical behavior that emerges from ladder diagrams underlies all of the critical properties encoded in the dynamical fluctuations and responses, as discussed in general terms in I.

\section{Dynamical responses}

Dynamical response functions, defined in Eq. (2), are particularly interesting because they provide, through inequalities such as Eq. (4), an estimate of the relevant dynamical fluctuations and because they are related to threepoint dynamical correlations, Eq. (3).

An exact expression for dynamical response functions can be derived noting that $G$ is obtained by setting $\partial \Gamma / \partial G$ $\equiv 0$. Differentiating this relation with respect to a conserved variable $\Psi$ one can easily derive the relation [see Eq. (60) of I]

$$
\chi_{\Psi}=\frac{\partial G}{\partial \Psi}=-\left[\frac{\partial^{2} \Gamma}{\partial G \partial G}\right]^{-1} \frac{\partial^{2} \Gamma}{\partial G \partial \Psi}=A^{-1} B,
$$

which is represented in Fig. 4 using MCT diagrams.

Since the derivative is taken with respect to the average value of one of the conserved degrees of freedom $\Psi$, the wave vector entering into the ladder diagrams is zero. As a consequence, the scaling of dynamical response functions is given by Eq. (9), setting $q=0$ :

$$
\begin{aligned}
& \sim \frac{B(q=0)}{\sqrt{\epsilon}} g_{\beta}\left(\frac{t}{\tau_{\beta}}\right), \quad t \propto \tau_{\beta}, \\
& \sim \frac{B(q=0)}{\epsilon} g_{\alpha}\left(\frac{t}{\tau_{\alpha}}\right), \quad t \propto \tau_{\alpha},
\end{aligned}
$$

where we have dropped the first argument of $g_{\beta}$, equal to zero here, and $B(q=0)$ reminds us that there is an additional contribution from the vertex $B$. We show below, see Eqs. (14)-(17), that these results can alternatively be obtained analytically using standard MCT results. However, the fieldtheoretical derivation shows more clearly the role of the ladder diagrams and is crucial to understand the relationship between dynamical response and dynamic fluctuations. We note that from the diagrammatic expression for $\chi_{\Psi}$ a clear relationship with three-point dynamical correlators appears. The diagrammatic expression of the correlation between the fluctuation of the dynamical structure factor and the fluctuation of conserved variables $\Psi$ reads (see I) $-A^{-1} B\{C$ $\left.-B^{\dagger} A^{-1} B\right\}^{-1}$, which contains the same diagrams as $\chi_{\Psi}$, with a propagator attached at the end. ${ }^{91}$

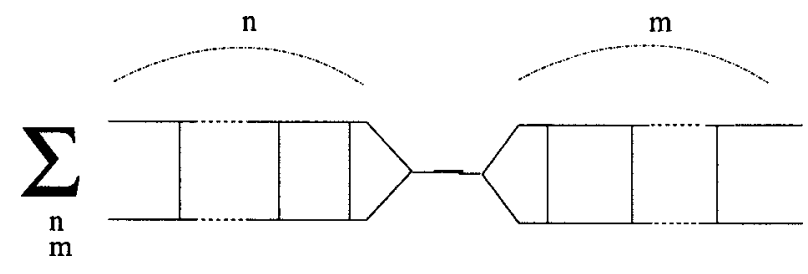

FIG. 5. Representation in terms of diagrams of $\left(A^{-1} B\right)\left\{C-B^{\dagger} A^{-1} B\right\}^{-1}$ $\left(A^{-1} B\right)^{\dagger}$, see Eq. (8), within MCT. It corresponds, roughly speaking, to "squaring" the ladders of Fig. 3.

In order to probe the spatial dependence of dynamical fluctuations related to ladder diagrams, zero-wave-vector response functions such as $\chi_{\Psi}(t)$ are not sufficient, and one should consider instead the response to a spatially modulated external field. ${ }^{31}$ It was recently proven in Ref. 31 that such a $q$-dependent dynamical response function has the same scaling as the one anticipated from ladder diagrams in Eq. (9).

\section{Ensemble and microscopic dynamics dependence of fluctuations}

In the following we illustrate, within MCT, the dependence on statistical ensembles and on microscopic dynamics of dynamical fluctuations which we have discussed in full generality in I. In particular, one finds that although $S_{4}(\mathbf{q}, t)$ and its $q \rightarrow 0$ limit are ensemble-independent quantities, $\chi_{4}(t)=S_{4}(\mathbf{q}=0, t)$ does depend on the ensemble and on microscopic dynamics. This fact reflects the subtle nature of global fluctuations when the thermodynamic limit is taken. ${ }^{22}$

Applying the general theory developed in I one finds that $S_{4}(\mathbf{q}, t)$ is given by the ladder diagrams in Fig. 3 plus "squared ladders," as shown in Fig. 5. The ladder diagrams shown in Fig. 5 are joined by a propagator at wave vector $\mathbf{q}$. In ensembles where all conserved degrees of freedom are fixed, e.g., Newtonian dynamics in the $N V E$ ensemble or Brownian dynamics in the $N V T$ ensemble, the propagator evaluated at $q=0$ vanishes, because conserved quantities do not fluctuate on the scale of the system size (and all propagators related to response fields are zero because they are proportional to $q$ at small $q$ ). Therefore, in these ensembles, simple ladder diagrams provide the sole contribution to $\chi_{4}(t)$ within MCT, causing $\chi_{4}(t)$ to scale as in Eq. (9) evaluated at $q=0$. This is also true for $p$-spin models, see Appendixes.

Instead, in ensembles where at least one conserved degree of freedom is allowed to fluctuate, e.g., the $N V T$ or $N P T$ ensembles for Newtonian dynamics, or the NPT ensemble for Brownian dynamics, the propagator joining the ladders in Fig. 5 does not vanish and contributes only to noncritical prefactors. In this case the diagrams corresponding to squared ladder diagrams dominate, at least close enough to the transition. Note that their overall scale might however be small, for example, if the compressibility or the specific heat are large or if the distance to the critical point become large. They lead to a modified critical behavior for $\chi_{4}(t)$ within MCT, reading 


$$
\begin{aligned}
& \frac{1}{\epsilon} \widetilde{g}_{\beta}\left(\frac{t}{\tau_{\beta}}\right), \quad t \propto \tau_{\beta}, \\
& \frac{1}{\epsilon^{2}} \widetilde{g}_{\alpha}\left(\frac{t}{\tau_{\alpha}}\right), \quad t \propto \tau_{\alpha},
\end{aligned}
$$

where $\widetilde{g}_{\alpha} \propto g_{\alpha}^{2}$ and $\widetilde{g}_{\beta} \propto g_{\beta}^{2}$.

These results provide nontrivial relationship between dynamical fluctuations in different ensembles and different microscopic dynamics. Although already suggested by the general theory developed in I they become sharp statements within MCT. For instance, the predicted dynamic scaling of $\chi_{4}(t)$ in the NVT ensemble for Newtonian dynamics is that of the squared ladder diagrams of Eq. (12), whereas the predicted scaling of $\chi_{4}(t)$ in the $N V T$ ensemble for Brownian dynamics is that of Eq. (11), which coincides with the one expected for Newtonian dynamics in the $N V E$ ensemble. Note that the critical mechanism underlying the dynamical fluctuations is the same for all ensembles and dynamics and is uniquely encoded in ladder diagrams. Indeed there is a unique length scale, diverging in the same way for all microscopic dynamics, which underlies the critical behavior. However, the coupling to conserved degrees of freedom may produce a large amplification of global fluctuations.

\section{Behavior of $S_{4}(q, t)$ and upper critical dimension}

The behavior of $S_{4}(\mathbf{q}, t)$ for $q \neq 0$ is apparently simpler because it does not depend on the statistical ensemble. Furthermore, all types of diagrams are present in its expression so that its qualitative critical behavior is independent of the microscopic dynamics, provided that at least density is locally conserved. However, since $S_{4}(\mathbf{q}, t)$ contains the two terms discussed previously (ladders and squared ladders) a crossover behavior might be expected. Although the squared ladders should dominate very close to $T_{c}$ they might become subdominant far from the critical point. Therefore one should be very cautious when comparing the present MCT predictions to the behavior of real liquids where the mode-coupling singularity $T_{c}$ is replaced by a smooth crossover towards an activated regime. In order to judge the relative importance of the two terms (ladder and squared ladder), one may focus on their $q=0$ value, which corresponds to $\chi_{4}^{N V E}$ for ladders and to $k_{B} T^{2} \chi_{T}^{2} / c_{V}$ for squared ladders. For example, in the case of the Lennard-Jones (LJ) mixture studied in I, the latter term becomes dominant only close to the transition $T \simeq 0.47$. As a consequence, for higher temperatures, the contribution of the squared ladders can be neglected and $S_{4}(q, t)$ will have the behavior presented in Eq. (9). A similar crossover is expected for $\chi_{4}(t)$, as confirmed numerically in I. The important difference with $S_{4}(\mathbf{q}, t)$ is that it is possible, at least in numerical simulations, to disentangle the different contributions to $\chi_{4}(t)$ by working in different ensembles.

Although four-point correlators were originally hoped to be suited to quantify precisely dynamical heterogeneities in glass formers, our results show that, although containing useful information on dynamical heterogeneities, they mix it with other, less interesting physical effects. This is a further motivation to study dynamical response to spatially modu- lated fields introduced in Ref. 31, for which only the simple ladder diagrams contribute and which, therefore, allows one to obtain clearer and more direct information on dynamic correlations. In future work, it would be extremely interesting to compare this response function computed within MCT (Ref. 31) to its direct numerical evaluation in a simulated liquid.

Finally we note that, within MCT, the scaling of dynamic fluctuations in the ensemble where all conserved variables fluctuate is different from the one predicted by BB. ${ }^{34}$ This implies that the upper critical dimension of the theory, found to be $d_{c}=6$ in Ref. 34 , has to be revised accordingly. Strictly speaking $d_{c}=6$ only applies for dynamics with no conserved variables, as for mean-field spin-glass models, but cannot be used for dynamics conserving at least one variable, such as density. Focusing on the $\beta$ regime, the fluctuations of the nonergodicity parameter in a region of size $\xi \sim \epsilon^{-1 / 4}$ grow as $\delta q \propto \xi^{4-d / 2}$, where $d$ is the space dimension. Imposing, in the spirit of a Ginzburg criterion, that $\delta q$ must be much smaller than the critical behavior of the order parameter, i.e., $q_{c}-q \sim \sqrt{\epsilon}$, one finds that fluctuations become dominant below the upper critical dimension $d_{c}=8$. In Ref. 44 it is shown that this result can be obtained from diagrammatic considerations: below $d_{c}=8$, corrections to MCT are found to diverge in the infrared regime.

\section{The $k$ dependence of dynamical fluctuations within MCT}

Several different definitions of $\chi_{4}(t)$ have been employed in the literature. ${ }^{12,14,15,18,34,45-48}$ Regardless of definition, since two-point density fluctuations must depend on the dynamically probed length scale $\left(\sim k^{-1}\right)$, the detailed behavior of $\chi_{4}(t)$ will also depend on this length scale, see, e.g., Ref. 48. Physically, the dependence of the fluctuations on length scale reflects the coupling or sensitivity of cooperative motion to behavior on the scale of the measured two-point fluctuations. For example, the expectation that high-frequency phonons do not couple strongly to the large length scale dynamic heterogeneity is reflected by the fact that a $\chi_{4}(t)$ that focuses on short length scales associated with vibrations cannot exhibit the sizable normalized peak values that are connected to large cooperative lengths (but see the discussion in Ref. 14). Only at a critical point would one expect all modes to couple in such a way that the behavior of $\chi_{4}(t)$ would exhibit truly universal properties. Since this issue cannot be discussed within $p$-spin models which contain no length scale (see Appendixes), we turn instead in this section to liquid state MCT which contains the complete wave vector dependencies of dynamic functions.

The dependence of $\chi_{4}(t)$ as a function of length scale was first discussed by Lačević et al., ${ }^{46}$ who used the definition

$$
\chi_{4}(t)=\frac{\beta V}{N^{2}}\left[\left\langle Q^{2}(t)\right\rangle-\langle Q(t)\rangle^{2}\right],
$$

where $N, V$, and $\beta$ are the number of particles, the volume, and the inverse temperature, respectively, and $Q(t)$ $=\sum_{i j} w\left(\left|\mathbf{r}_{i}(0)-\mathbf{r}_{j}(t)\right|\right)$ and $w\left(\left|\mathbf{r}_{1}-\mathbf{r}_{2}\right|\right)=1$ for $\left|\mathbf{r}_{1}-\mathbf{r}_{2}\right| \leqslant a$ and is 
zero otherwise. Here $a$ is a cutoff parameter. Lačević et al. explicitly showed that within this definition of $\chi_{4}(t)$, the value of $a$ that maximizes the peak height is close to the global Debye-Waller amplitude of the mean-squared displacement. ${ }^{46}$ For values of the cutoff that are larger or smaller, the absolute amplitude of $\chi_{4}(t)$ decreases. It can be noted in this work that the shape of $\chi_{4}(t)$ is sensitive to the value of $a$ as well, although no systematic study of this dependence was investigated.

Using a different definition of $\chi_{4}(t)$, namely, that defined by Eq. (50) below, Dauchot et al. noted that for a weakly sheared granular system, the slope of $\chi_{4}(t)$ increases as the wave vector decreases. ${ }^{18}$ This particular dependence has been studied in more detail in a recent work. ${ }^{48}$ From both molecular dynamics simulation and the direct analysis of a class of kinetic facilitated models, Chandler et al. have detailed the length scale dependence of a variety of definitions of $\chi_{4}(t)$ and have argued that a generic feature of this dependence is that the growth of $\chi_{4}(t)$ to its peak becomes significantly more rapid as the intrinsic length scale increases. It was also $\operatorname{argued}^{48}$ that this result is inconsistent with the predictions of mode-coupling theory outlined in Ref. 14. Since the $k$-dependent $\chi_{4}(t)$ in facilitated models has been discussed in detail in Ref. 48, we focus below on the predictions of mode-coupling theory. In particular, we show that, despite statements to the contrary, mode-coupling theory is at least in qualitative accord with the behavior found from computer simulation, as detailed in Ref. 48.

An important aspect of the physical content of the $k$ dependence of $\chi_{4}(t)$ is embodied in the consideration of the distinction between $\beta$ and $\alpha$ relaxations, and the implication that this distinction holds for the length scales of dynamic heterogeneity. At a given density and temperature, a welldefined plateau in the two-point density correlator for wave vectors near the first diffraction peak in the static structure factor $S(k)$ will saturate as $k$ is decreased. Eventually, as $k$ is decreased further the hydrodynamic regime is reached, where the local cooperative processes associated with dynamic heterogeneity are averaged out. At a fixed distance from the dynamical transition temperature $T_{c}$, as $k$ is decreased first the $\beta$-relaxation window decreases in duration. ${ }^{49}$ Concomitantly, the stretching exponent of the $\alpha$ relaxation increases continuously. Eventually, the crossover is complete when the $\beta$ window is no longer observable, and the stretching exponent saturates at unity, signifying long-time hydrodynamic behavior.

The theoretical considerations made in Ref. 14 are based on the asymptotic predictions of mode-coupling theory. Arbitrarily close to $T_{c}$ and in systems for which $T_{c}$ is not avoided, the predictions of Ref. 14 are nearly universal in the sense that the effects mentioned above are only seen in the strict $k \rightarrow 0$ limit. By considering the $k$ dependence of induced susceptibility $\chi_{x}(t)$ for a fixed, finite distance from $T_{c}$ one gains a qualitative understanding of how the universal features expected for $k$ near the first diffraction peak of $S(k)$ are changed as $k$ decreases.

As discussed in the previous sections dynamical fluctuations encoded in ladder diagrams are visible either in $\chi_{4}(t)$ or in the dynamical response. However, dynamical responses are accessible to direct quantitative numerical computations that are an essential tool in order to discuss the crossover issues discussed above. Let us now, for completeness and clarity, rederive the results for dynamical responses using only standard MCT results ${ }^{27}$ being particularly careful about the $k$ dependence.

In the $\alpha$ regime close to the transition, but still in the liquid phase, the dynamical structure factor scales like $F(k, t) \simeq f_{\alpha}^{k}\left(t / \tau_{\alpha}(\epsilon)\right)$, where $\tau_{\alpha}=\epsilon^{-\gamma}$ and $\gamma=1 / 2 a+1 / 2 b$. Thus, we find that in the $\alpha$ regime $\chi_{x}(k, t)=\partial F(k, t) / \partial x$ reads

$$
\chi_{x}(k, t) \simeq \frac{1}{\epsilon} g_{\alpha}^{k}\left(t / \tau_{\alpha}\right), \quad g_{\alpha}^{k}(x)=-\frac{x}{\gamma} \frac{d f_{\alpha}^{k}}{d x} .
$$

We temporarily change notation to emphasize the $k$ dependence, namely, we promote $\chi_{x}(t)$ to $\chi_{x}(k, t)$. In the $\beta$ regime and close to the transition $F(k, t) \simeq S(k) q(k)$ $+S(k) h(k) \sqrt{\epsilon} f_{\beta}\left(t / \tau_{\beta}\right)$, where $\tau_{\beta}=\epsilon^{-1 / 2 a}, q(k)$ is the nonergodic parameter and $h(k)$ is the critical amplitude. Thus, we find that in the $\beta$ regime $\chi_{x}(k, t)$ reads

$$
\begin{aligned}
& \chi_{x}(k, t) \simeq \frac{h(k) S(k)}{\sqrt{\epsilon}} g_{\beta}\left(t / \tau_{\beta}\right), \\
& g_{\beta}(x)=-f_{\beta}(x)-\frac{x}{2 a} f_{\beta}^{\prime}(x) .
\end{aligned}
$$

Analyzing the $\beta$ regime with a large but not diverging time and matching the $\alpha$ regime with the $\beta$ regime impose constraints on the large and small $x$ behaviors of $g_{\beta}(x)$ and $g_{\alpha}^{k}(x)$. Requiring that in the early $\beta$ regime the $\epsilon$ dependence should drop out of $\chi_{x}(k, t)$, we find that

$$
\chi_{x}(k, t) \sim t^{a}, \quad 1 \ll t \ll \tau_{\beta} .
$$

Analogously, matching the $\alpha$ and $\beta$ regimes leads to

$$
\chi_{x}(k, t) \sim \epsilon^{(b-a) / 2 a} t^{b}, \quad \tau_{\beta} \ll t \ll \tau_{\alpha},
$$

interpolating between $\chi_{x} \sim \epsilon^{-1 / 2}$ for $t=\tau_{\beta}$ and $\chi_{x} \sim \epsilon^{-1}$ for $t$ $=\tau_{\alpha}$, before decaying back to zero for $t \gg \tau_{\alpha}$.

All these results are valid close enough to the transition but, as discussed previously, we expect crossovers as functions of $k$ and time. In order to study this issue numerically, we solve the full, wave-vector-dependent mode-coupling equations for the self-intermediate function $F_{s}(k, t)$ for a dense colloidal suspension, which are directly coupled to the collective density fluctuations $F(k, t)$ as

$$
\frac{\partial F_{s}(k, t)}{\partial t}+D_{0} k^{2} F_{s}(k, t)+\int_{0}^{t} d t^{\prime} M_{s}\left(k, t-t^{\prime}\right) \frac{\partial F_{s}\left(k, t^{\prime}\right)}{\partial t^{\prime}}=0,
$$

where $D_{0}$ is the bare diffusion constant, and $M_{s}(k, t)$ is the self-memory function that can be expressed as

$$
M_{s}(k, t)=\frac{\rho_{0} D_{0}}{(2 \pi)^{3}} \int d \mathbf{k}^{\prime}\left\{\hat{\mathbf{k}} \cdot \mathbf{k}^{\prime} c\left(k^{\prime}\right)\right\}^{2} F_{s}\left(\left|\mathbf{k}-\mathbf{k}^{\prime}\right|, t\right) F\left(k^{\prime}, t\right) .
$$

Here, $\rho_{0}=N / V$ is the number density, $\hat{\mathbf{k}}=\mathbf{k} /|\mathbf{k}|, c(k)$ is the direct correlation function, and $\rho_{0} c(k)=1-1 / S(k)$. These 


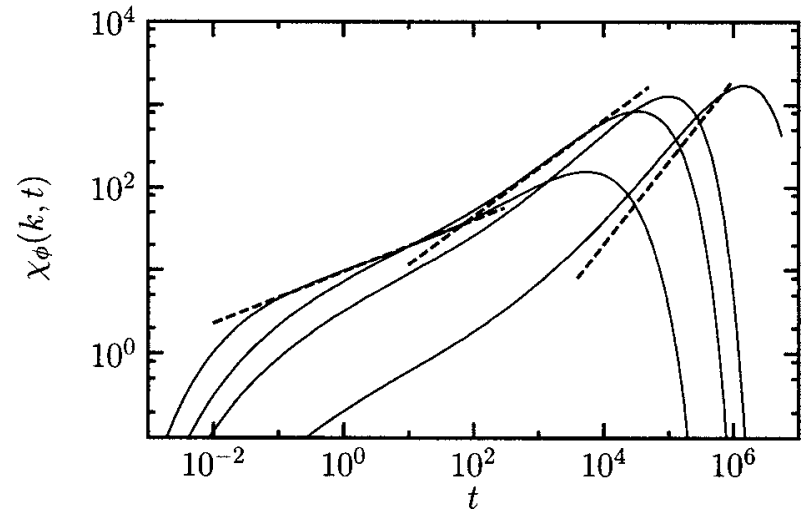

FIG. 6. Dynamic susceptibility $\chi_{\phi}(k, t)$, Eq. (22), predicted by MCT for hard spheres at fixed volume fraction above the glass transition, $\phi_{c}-\phi$ $=10^{-3}$ for various wave vectors from $k=19.35$ to $k=0.75$ (in particle size units) from left to right. Power laws for the largest $k$ are the asymptotic results $t^{a}$ and $t^{b}$ with $a=0.312$ and $b=0.583$, while $\chi_{\phi}(t) \sim t$ describes well the data at small $k$ (rightmost curve).

equations are solved using as input the solution of the modecoupling equations for the full density fluctuations

$$
\frac{\partial F(k, t)}{\partial t}+\frac{D_{0} k^{2}}{S(k)} F(k, t)+\int_{0}^{t} d t^{\prime} M\left(k, t-t^{\prime}\right) \frac{\partial F\left(k, t^{\prime}\right)}{\partial t^{\prime}}=0,
$$

where

$$
M(k, t)=\frac{\rho_{0} D_{0}}{(2 \pi)^{3}} \int d \mathbf{k}^{\prime}\left|V\left(\mathbf{k}, \mathbf{k}^{\prime}\right)\right|^{2} F\left(\left|\mathbf{k}-\mathbf{k}^{\prime}\right|, t\right) F\left(k^{\prime}, k\right),
$$

and $V\left(\mathbf{k}, \mathbf{k}^{\prime}\right)=\hat{\mathbf{k}} \cdot \mathbf{k}^{\prime} c\left(k^{\prime}\right)+\hat{\mathbf{k}} \cdot\left(\mathbf{k}-\mathbf{k}^{\prime}\right) c\left(\left|\mathbf{k}-\mathbf{k}^{\prime}\right|\right)$. These equations are solved for a model hard sphere suspension with input from $S(k)$ calculated from the Percus-Yevick closure at various volume fractions $\phi$. The wave vector cutoff is taken to be $k_{c}=50$ in units of the particle size, and the grid number is taken to be 100 . The equations of motion are integrated with the algorithm of Fuchs et al. ${ }^{49}$ The induced susceptibility

$$
\chi_{\phi}(k, t)=\frac{\partial F_{s}(k, t)}{\partial \phi}
$$

is computed via numerical differentiation. In Fig. 6 the induced susceptibility is shown for different values of wave vector from those higher than the first peak in $S(k)$ to those significantly below. The behavior of $\chi_{\phi}(k, t)$ for $k$ close to the first diffraction peak displays the two power law regimes described in Sec. II. When $k$ is decreased the power law describing how $\chi_{\phi}(k, t)$ reaches its peak clearly shows an increasing value. This behavior is qualitatively compatible with the one discussed theoretically and found in numerical simulations of KCM's and atomistic liquids in Ref. 48 and experiments on granular materials. ${ }^{18}$ It makes clear that corrections to the critical behavior are different depending on $k$. In particular, the limit $k \rightarrow 0$ and $T \rightarrow T_{c}$ clearly do not commute.

Note that, although the critical behavior is expected to be the same for $\chi_{\phi}(k, t)$ and $\chi_{4}(t)$ in the $N V E$ ensemble, the corrections to the critical scaling might not be the same. However, the same qualitative considerations regarding time and wave vector dependencies should hold. Furthermore, using the bounds derived in I the present results yield direct quantitative predictions for the behavior of the dynamic susceptibility $\chi_{4}(t)$ in the hard sphere system since its scaling behavior is the same as to the one of $\chi_{\phi}^{2}(k, t)$, which obviously follows from Eqs. (14)-(17).

\section{DYNAMIC SUSCEPTIBILITY AND DIVERGING LENGTH SCALES IN KCMS}

\section{A. Models and observables}

In this section we proceed with the computation of the dynamic susceptibility $\chi_{T}(t)$ and its comparison to the previously studied $\chi_{4}(t)$ in KCMs. ${ }^{28}$ Our motivation here stems from the fact that the study of KCMs has greatly contributed to our present understanding of the dynamically heterogeneous dynamics of supercooled liquids. ${ }^{13,14,28,47,48,50-56}$ Moreover, the variety of available models allows one to grasp the variety of possible behaviors that could possibly be encountered in real materials. Finally the relative simplicity of the models makes them suitable to large scale numerical simulations, which might help data analysis in real materials, while scaling laws and exact results can be obtained by standard theoretical tools of statistical mechanics.

KCMs are spin models (lattice gas versions also exist ${ }^{57}$ ) generically described by a simple, usually noninteracting Hamiltonian, and a set of dynamic rules with nontrivial constraints forbidding some of the transitions and therefore making the overall dynamics glassy. In the following we will focus on spin models characterized by the FredricksonAndersen (FA) Hamiltonian ${ }^{51}$

$$
H=\sum_{i=1}^{N} n_{i}
$$

where $n_{i}=0,1$ is a binary variable defined on each point of a hypercubic lattice in dimension $d$. Physically, $n_{i}=0 \quad\left(n_{i}=1\right)$ represents a site $i$ which is immobile (mobile), and has therefore an energy which is smaller (larger) than the average energy, given by

$$
\left\langle n_{i}\right\rangle=c(T)=\frac{1}{1+e^{1 / T}} .
$$

The spins evolve with a single spin flip dynamics, so that the model dynamics is entirely defined by the transition rates between states 1 and 0 ,

$$
n_{i}=0 \underset{\mathcal{C}_{i}}{\stackrel{\mathcal{C}_{i} c}{\rightleftarrows}} n_{i}=1
$$

where $\mathcal{C}_{i}$ is a kinetic constraint on site $i$ which can become 0 depending on the local environment of site $i$, therefore prohibiting some specific transitions. We shall study in detail two different spin facilitated models where the kinetic constraint takes the following forms: 


$$
\mathcal{C}_{i}=1-\prod_{j}\left(1-n_{j}\right)
$$

and

$$
\mathcal{C}_{i}=1-\prod_{j, k}\left(1-n_{j} n_{k}\right),
$$

for one- and two-spin facilitated models, respectively. In the expressions for $\mathcal{C}_{i}$ the products are over nearest neighbors of site $i$. The constraints, respectively, become equal to 1 when, respectively, at least one or two of their nearest neighbors is mobile, therefore capturing the idea of dynamic facilitation: mobile regions locally favor the creation of more mobility. ${ }^{13,51}$

Due to the presence of a heat bath, the dynamics of KCMs do not conserve energy. Physically this means that heat can be locally provided to a spin to allow the creation of a mobility excitation without the need to borrow energy from the neighboring sites. In principle the results obtained from $\mathrm{KCMs}$ should then be compared to the $N V T$ dynamics of molecular liquids. As opposed to the MCT results described above, no prediction can be made from KCMs concerning the role of a conservation law for the energy. For kinetically constrained lattice gases, however, a quantitative comparison between spontaneous fluctuations, $\chi_{4}(t)$, and fluctuations induced by a change of density, $\chi_{\rho}(t)$, can be performed. ${ }^{48}$

A second important consequence of the presence of a heat bath is that neither the fluctuation-dissipation relation in Eq. (3) nor the inequality [Eq. (4)] applies to KCMs, and we are therefore left with three independent dynamic quantities, namely,

$$
\begin{aligned}
& \chi_{T}(t)=\frac{\partial\langle P(t)\rangle}{\partial T}, \\
& C_{P E}(t)=N\langle\delta P(t) \delta e(0)\rangle, \\
& \chi_{4}(t)=N\left\langle\delta P^{2}(t)\right\rangle,
\end{aligned}
$$

where we have defined the instantaneous value of the energy density,

$$
e(t)=\frac{1}{N} \sum_{i=1}^{N} n_{i}(t) .
$$

Following earlier works on KCMs we choose to work with the persistence function as the relevant two-time dynamical object,

$$
P(t)=\frac{1}{N} \sum_{i=1}^{N} P_{i}(t),
$$

where $P_{i}(t)$ denotes the persistence of the spin $i$ between times 0 and $t$. Its thermodynamic average, $\langle P(t)\rangle$, has recently been the subject of a number of theoretical studies. ${ }^{13,47,54,58-60}$ Note that for KCMs, the Cauchy-Schwarz inequality could still be of some use. For the present variables this leads to

$$
\chi_{4}(t) \geqslant \frac{C_{P E}^{2}(t)}{T^{2} c_{V}},
$$

where $c_{V}(T)=d c / d T=e^{1 / T} /\left[T^{2}\left(1+e^{1 / T}\right)^{2}\right]$ is the specific heat. The main difference between the inequalities (31) and (4) is that the right hand side of Eq. (31) is given by a correlation function which is not easily accessible in experiments, contrary to the susceptibility $\chi_{T}(t)$ appearing in Eq. (4). Of course, $C_{P E}(t)$ can be measured in numerical experiments, as shown below.

At the level of the spatial correlations, two distinct correlators also need to be studied:

$$
\begin{aligned}
& S_{T}(r, t)=\left\langle\delta P_{i}(t) \delta n_{i+r}(0)\right\rangle, \\
& S_{4}(r, t)=\left\langle\delta P_{i}(t) \delta P_{i+r}(t)\right\rangle .
\end{aligned}
$$

Their Fourier transforms, $S_{4}(q, t)$ and $S_{T}(q, t)$, can equivalently be studied, and it is obvious that $S_{4}(q=0, t)=\chi_{4}(t)$ and $S_{T}(q=0, t)=C_{P E}(t)$, these quantities representing the volume integrals of the spatial correlations $S_{4}(r, t)$ and $S_{T}(r, t)$, respectively.

\section{B. Results for one-spin facilitated FA models}

The one-spin facilitated FA model has been studied numerically and analytically in various spatial dimensions in much detail. ${ }^{13,14,28,47,48,54,58-60}$ These studies have shown that the model exhibits dynamic heterogeneity and large spontaneous fluctuations of the two-time dynamics, although relaxation time scales grow only in an Arrhenius fashion as temperature is decreased,

$$
\tau_{\alpha} \sim c^{-\Delta} \sim \exp (\Delta / T),
$$

with $\Delta=3$ for $d=1$ and $\Delta=2$ for $d>2$. Interestingly, these works suggest that even strong material should display dynamic heterogeneity. This was confirmed by simulations ${ }^{61}$ and experiments ${ }^{62}$ which reported deviations from the Stokes-Einstein relation, although the FA model itself presents no such deviations for $d \geqslant 2$.

As usual, the four-point susceptibility $\chi_{4}(t)$ is found to have nonmonotonic time dependence. Therefore it shows a peak, $\chi_{4}^{\star}(T)=\chi_{4}\left(t \sim \tau_{\alpha}\right)$, whose position shifts to larger times and whose height increases when temperature decreases. One finds dynamic scaling ${ }^{13,47,58}$

$$
\chi_{4}^{\star} \sim c^{-\gamma} \sim \exp (\gamma / T) \sim \tau_{\alpha}^{\gamma / \Delta},
$$

with $\gamma=1$ in all spatial dimensions. The corresponding spatial dynamic correlations have also been studied. Analytically, one can compute these quantities approximately by making the assumption that the system can be described as an assembly of defects which diffuse independently with diffusion constant $D=c$. This was called "independent defect approximation" in Ref. 14. In three dimensions, one finds

$$
S_{4}(q, t) \approx \chi_{4}(t) \mathcal{S}_{4}\left[q^{2} \xi_{4}^{2}(t)\right],
$$

with a diffusively growing length scale,

$$
\xi_{4}(t)=\sqrt{c t},
$$

and the scaling function 


$$
\mathcal{S}_{4}(x)=2 \frac{x-1+e^{-x}}{x^{2}} .
$$

Additionally the four-point dynamic susceptibility behaves as follows:

$$
\chi_{4}(t) \approx \frac{c_{2}}{2 c}\left(\frac{t}{\tau_{\alpha}}\right)^{2} \exp \left(-\frac{2 t}{\tau_{\alpha}}\right),
$$

with $c_{2}$ a numerical factor. These predictions are in good agreement with direct simulations of the FA model, the only discrepancy being that the scaling function for $S_{4}(q, t)$ shows deviations from its $1 / q^{2}$ predicted large $q$ behavior when times become very large, $t \gg \tau_{\alpha}$.

The computation of $\chi_{T}(t)$ is easy given that the average persistence obeys time temperature superposition, $\langle P(t)\rangle$ $=f\left(t / \tau_{\alpha}\right)$, the scaling function $f(x)$ being well described, for times which are not too long, ${ }^{60}$ by a stretched exponential form, $f(x)=\exp \left(-x^{\beta}\right)$, with $\beta=1 / 2$ for $d=1$ and $\beta=1$ for $d>2$. Therefore one immediately gets

$$
\chi_{T}(t)=-\frac{\Delta \beta}{T^{2}}\left(\frac{t}{\tau_{\alpha}}\right)^{\beta} \exp \left[-\left(\frac{t}{\tau_{\alpha}}\right)^{\beta}\right] .
$$

This shows that $\chi_{T}(t)$ displays a nonmonotonic time dependence with a peak arising at time $t \sim \tau_{\alpha}$, diverging as $\chi_{T}^{\star} \sim-1 / T^{2}$ when $T$ goes to zero. Finally, the behavior of $\chi_{T}(t)$ before the peak, $t \ll \tau_{\alpha}$, is a power law, $\chi_{T}(t) \sim t^{\beta}, \beta$ being the value of the stretching exponent characterizing also the $\alpha$ relaxation.

If one considers the quantity $T^{2} \chi_{T}^{2} / c_{V}$ appearing in the inequality (4), one finds at the peak,

$$
\frac{T^{2}}{c_{V}}\left(\chi_{T}^{\star}\right)^{2} \sim c^{-1} \sim \exp (1 / T) \sim \chi_{4}^{\star},
$$

so that both sides of the inequality (4) have similar scaling properties at low temperatures in this model. This is not an obvious result given that these quantities are not related by the thermodynamic relations and inequalities outlined in Sec. I.

Notice, however, that this similarity appears coincidental because the whole divergence of the first term in Eq. (41) is due to the very strong temperature dependence of the specific heat at low temperature which itself results from the noninteracting FA Hamiltonian (23). In real materials, the specific heat is almost temperature independent when the glass transition is approached and the growth of the term $T^{2} \chi_{T}^{2} / c_{V}$ is mainly due to the growing susceptibility $\chi_{T}(t)$ itself.

Following steps similar to those described in Ref. 14 it is possible to compute both the correlator in Eq. (32) and its volume integral in Eq. (28) within the independent defect approximation. In three dimensions, one finds for $S_{T}(q, t)$ a scaling form very similar to Eq. (36),

$$
S_{T}(q, t) \approx C_{P E}(t) \mathcal{S}_{T}\left[q^{2} \dot{\xi}_{T}^{2}(t)\right],
$$

with

$$
C_{P E}(t) \approx c_{1}\left(\frac{t}{\tau_{\alpha}}\right) \exp \left(-\frac{c_{1} t}{\tau_{\alpha}}\right),
$$

where $c_{1}$ is a numerical factor, the corresponding correlation length scale

$$
\xi_{T}(t)=\sqrt{c t},
$$

and the scaling function

$$
\mathcal{S}_{T}(x)=\frac{1-e^{-x^{2}}}{x^{2}} .
$$

These calculations show that, within one-spin facilitated models, the physical content of the correlators $S_{4}$ and $S_{T}$ is essentially the same. Physically, this is because two sites are dynamically correlated, and therefore contribute to $S_{4}(r, t)$, if they are visited by the same diffusing mobility defect. Similarly, two sites contribute to $S_{T}(r, t)$ if one of them contains at time 0 the first defect which will visit the second one for $t$ $>0$. This implies that the correlation length scales $\xi_{4}$ and $\xi_{T}$ both reflect the simple activated diffusion of point defects and therefore contain the same physical information; Eqs. (37) and (44) show that they are indeed equal. Additionally, the spatial correlators $S_{4}(q, t)$ and $S_{T}(q, t)$ are found to differ in their detailed expression, Eqs. (38) and (45), but they have the same asymptotic behaviors, $S_{T}\left(q \xi_{T} \ll 1\right) \sim$ const and $S_{T}\left(q \xi_{T} \gg 1\right) \sim 1 / q^{2}$, reminiscent of an Ornstein-Zernike form.

An additional piece of information derived from Eqs. (40) and (43) is the similar time dependence and scaling with temperature found for the quantities $T^{2} \chi_{T}(t)$ and $C_{P E}(t)$, despite the fact a fluctuation-dissipation relation such as Eq. (3) does not hold. Numerically we indeed find that both terms quantitatively differ, although merely by a numerical factor.

The independent defect approximation is thought to be a good representation of the one-spin facilitated model above its critical dimension, $d>2$, as confirmed by our numerical simulations in $d=3$. In Fig. 7, we provide additional numerical evidence that these findings are correct in $d=1$ as well. The top figure shows that the time dependence and scaling with temperature of three different quantities, $\chi_{4}(t)$, $C_{P E}^{2}(t) /\left(T^{2} c_{V}\right)$, and $T^{2} \chi_{T}^{2}(t) / c_{V}$, are the same. Moreover, the Cauchy-Schwarz inequality (31) is satisfied by our numerics, as it should be, while the inequality (4) derived for Newtonian dynamics is found to be violated by a factor which is about 2 at the peak. The bottom panel in Fig. 7 shows $S_{4}\left(q, \tau_{\alpha}\right)$ and $S_{T}\left(q, \tau_{\alpha}\right)$ for the FA model in $d=1$. As predicted from the independent defect approximation, both correlation functions are slightly different in their shape but share a common behavior, a plateau at small $q$ and a $1 / q^{2}$ decay at large $q$. We have checked that the equivalence $\xi_{4}(t) \sim \xi_{T}(t)$ also holds in numerical simulations, confirming that dynamic-dynamic and dynamic-energy spatial correlations are essentially equivalent quantities in the context of noncooperative KCMs. This is physically expected since by definition of the kinetic constraints in Eq. (26), it is those regions with high potential energy which trigger the dynamics of the nearby sites: this is the essence of the dynamic facilitation idea. 

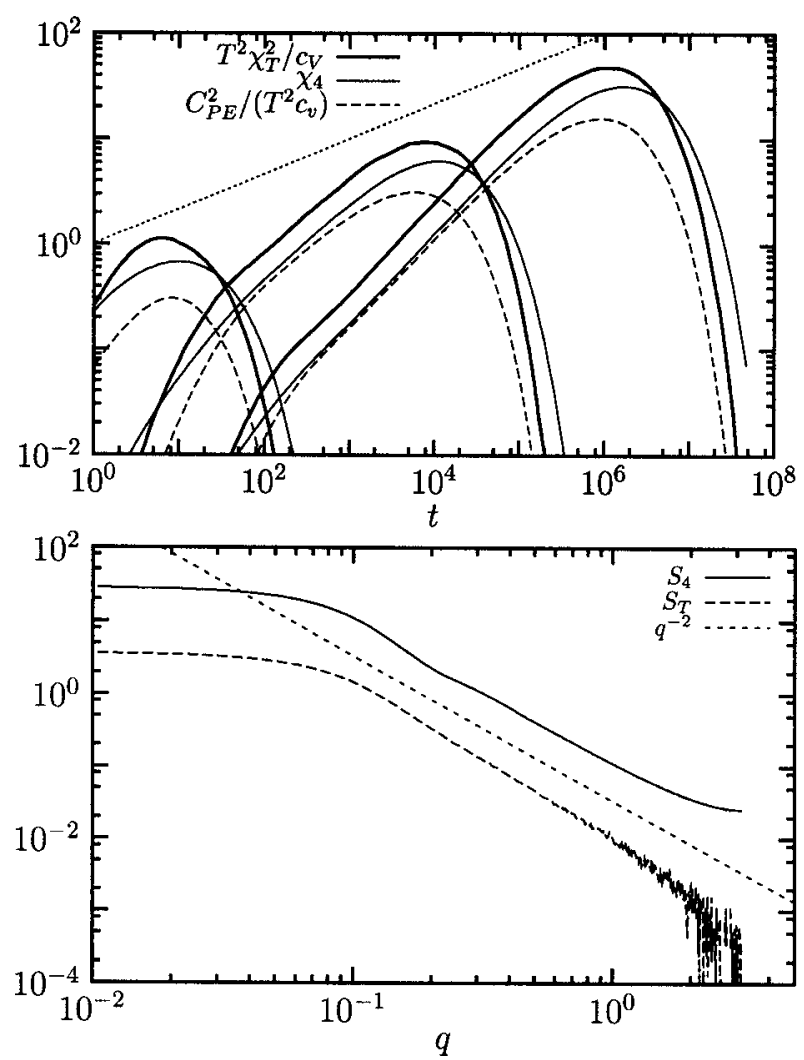

FIG. 7. Dynamic susceptibilities and spatial correlations in the one-spin facilitated FA model in one dimension. Top: Various dynamical susceptibilities are shown as a function of time for temperatures $T=1.0,0.5$, and 0.2 (from left to right). They behave similarly with time, and their peak scales as $\tau_{\alpha}^{\gamma / \Delta}=\tau_{\alpha}^{1 / 3}$, as shown with dots. Bottom: Dynamic structure factors $S_{4}(q, t)$ and $S_{T}(q, t)$ at time $t=\tau_{\alpha}$ for $T=0.3$. Both functions behave as a constant at small $q$, and as $1 / q^{2}$ at large wave vectors, as shown with a dashed line.

We conclude this section on one-spin facilitated models by briefly discussing the case of the East model, ${ }^{50}$ which is defined with the same FA Hamiltonian (23) and is also a one-spin facilitated model where the kinetic constraint is defined similar to Eq. (26), the only difference being that the product appearing in Eq. (26) is now restricted to only one neighbor in each spatial direction. This "hyper-East" model was called the North or East or Front model in $d=3 .{ }^{63}$ This directionality of the constraint makes the dynamics of the East model slower than that of the FA model, and relaxation time scales now grow in a super-Arrhenius fashion, so that the exponent $\Delta$ appearing in Eq. (34) becomes temperature dependent, $\Delta(T) \sim-\ln c(T)$. The East model is therefore a $\mathrm{KCM}$ for fragile glasses. Additionally, time temperature superposition does not hold. Relaxation is still described by stretched exponentials but the stretching exponent is also temperature dependent, with $\beta(T) \sim T$ at low temperature. $^{64,65}$ Despite these qualitative differences between strong and fragile models, our main conclusions still hold. The three dynamic susceptibilities shown in Fig. 7 also track each other, and this is again the result of subtle compensations between the scaling of correlation functions and the strong temperature dependence of the defect concentration. Similarly, the two different length scales $\xi_{4}$ and $\xi_{T}$ also bear the same physical content, although they now grow subdiffusively with time. ${ }^{14}$ This subdiffusive behavior affects the approach of the dynamic susceptibilities to their maximum. In the $d=1$ East model, one finds that before the peak $\chi_{T}(t) \sim t^{b(T)}$, where the exponent $b(T) \approx \beta(T)$ should decrease slowly when $T$ decreases. We find numerical values $b$ $\approx 0.2-0.4$ in the time window of our Monte Carlo simulations, where relaxation time scales increase from $\tau_{\alpha} \sim 10^{4}$ to $\tau_{\alpha} \sim 10^{8}$.

\section{Results for a two-spin facilitated FA model}

By comparison with one-spin facilitated models, much less is known about the behavior of two-spin facilitated models, because relaxation does not proceed by activated diffusion (or even subdiffusion) of point defects. ${ }^{51,52}$ In some cases, asymptotic mechanisms have been described which show that relaxation time scales grow very rapidly when temperature is decreased, although no finite temperature divergence is found. ${ }^{52,55}$ In these mechanisms, relaxation occurs via the diffusion of "superdefects" whose concentration decreases when $T$ decreases and whose size is itself a decreasing function of temperature. For this reason these models are sometimes called "cooperative KCMs." Very recently, a KCM was specifically engineered to yield an example of a finite temperature singularity, but we do not discuss this example further. ${ }^{66}$

An additional point of theoretical interest of cooperative $\mathrm{KCMs}$ is that, when studied on Bethe lattices, they display a dynamical transition at finite temperature which is reminiscent of the mode-coupling singularity described in Sec. II. Moreover, dynamic fluctuations can be studied in some analytic detail in the Bethe limit, while no analytic study of dynamic fluctuations on finite dimensional lattices is available.

We now focus on the two-spin facilitated model in dimension $d=2$, the "22FA model," as a specific example of a cooperative model. Our choice is motivated by the relatively large number of earlier studies dedicated to this model, ${ }^{51,52,55}$ the fact that its Bethe limit was also considered ${ }^{56}$ and that it is sufficiently far from its mean-field limit that deviations from mean-field behavior are clearly observed. It was indeed realized early on that the model does not display a power law divergence of its relaxation time at finite $T,{ }^{51,52}$ contrary to more constrained models where numerics seemed to indicate the presence of a mean-field-like singularity, ${ }^{57}$ now discarded. ${ }^{55,67}$

Adapting the general results of Ref. 55 to the specific example of the 22FA model, we expect the following scaling results. The relaxation time grows as

$$
\tau_{\alpha} \sim \exp \left(\frac{a}{c}\right) \sim \exp \left[a \exp \left(\frac{1}{T}\right)\right],
$$

where $a$ is a numerical factor. The double exponential divergence makes the 22FA a very fragile glass-former model.

The scaling of the four-point dynamic susceptibility is obtained as follows. At a given temperature, relaxation occurs via the diffusion of superdefects of size $\ell(T)$. By coarse graining the system up to size $\ell$, relaxation then resembles the diffusion of independent defects, and the results of the independent defect approximation can be carried out. There- 
fore, we expect $\chi_{4}^{*} \sim c_{\ell}^{-1}$, where $c_{\ell}$ is the concentration of superdefects. Using the results of Ref. 55, we get

$$
\chi_{4}^{\star} \sim \exp \left(\frac{a}{c}\right) \sim \tau_{\alpha} .
$$

We evaluate the leading divergence of $\chi_{T}(t)$ by assuming time temperature superposition, i.e., $\chi_{T}(t)=\partial f\left(t / \tau_{\alpha}\right) / \partial T$. Using Eq. (46) we get $\chi_{T}^{*} \sim \exp (a / c) /\left(T^{2} c\right)$, up to an irrelevant numerical prefactor. As a consequence, the right-hand side of the inequality (4) scales as

$$
\frac{T^{2}}{c_{V}}\left(\chi_{T}^{\star}\right)^{2} \sim c^{-3} \sim\left(\ln \tau_{\alpha}\right)^{3} .
$$

By comparing Eqs. (48) and (47), we conclude that the dynamic heterogeneity quantified through $\chi_{4}(t)$ and $T^{2} \chi_{T}^{2}(t) / c_{V}$ is very different, since $\chi_{4}(t)$ is predicted to diverge as a power of $\tau_{\alpha}$, while the term involving $\chi_{T}$ should diverge only logarithmically with $\tau_{\alpha}$. For cooperative models, the "coincidental" compensation due to the specific heat arising in noncooperative model is not effective.

Since these results are expected to hold only very close to $T=0$, we have performed numerical simulations of the 22FA model. In these Monte Carlo simulations, we cover the temperature regime $T=2.6$ down to $T=0.43$, which corresponds to about 7 decades of relaxation time scales. In this temperature window, $\tau_{\alpha}$ cannot be fitted with an inverse power law $\tau_{\alpha} \sim\left(T-T_{c}\right)^{-\alpha}$ as in the Bethe limit, showing that strong non-mean-field effects are indeed present. However, the form (46) is not completely successful either, suggesting that the true asymptotic regime is beyond the realm of numerical simulations (see Ref. 68 for a discussion of this point in a similar context) and that the numerical regime lies somewhat in a crossover regime.

In Fig. 8 we compare the evolution of the peak of $\chi_{4}(t)$ and the corresponding peak in $T^{2} \chi_{T}^{2}(t) / c_{V}$ for the entire temperature range we have been able to access numerically. Quite strikingly we find that both functions scale very similarly on the whole temperature range. A similar result was recently reported for a cooperative constrained lattice gas in two dimensions. ${ }^{48}$ This similarity holds also at the level of the whole time dependence (not shown). From numerical simulations only, we would therefore conclude that the coincidence between the two terms already found for noncooperative models also applies in cooperative models. This numerical evidence is contradicted by the asymptotic analytic arguments given above. A possible way to reconcile these results is to assume that the temperature regime we have studied in the simulations is still too close to the mean-field Bethe lattice limit, where the scaling $\chi_{4} \sim T^{2} \chi_{T}^{2} / c_{V}$ is indeed expected to hold. This argument is, however, clearly weakened by the fact that many observables (time scales, persistence functions, and others, see Ref. 69) show visible deviations from their mean-field limit in the same temperature regime.

In the bottom panel of Fig. 8, we also show the comparison of the spatial correlations (32) and (33) measured in Fourier space. Whereas both correlators were found to be very similar for noncooperative models, numerics clearly re-
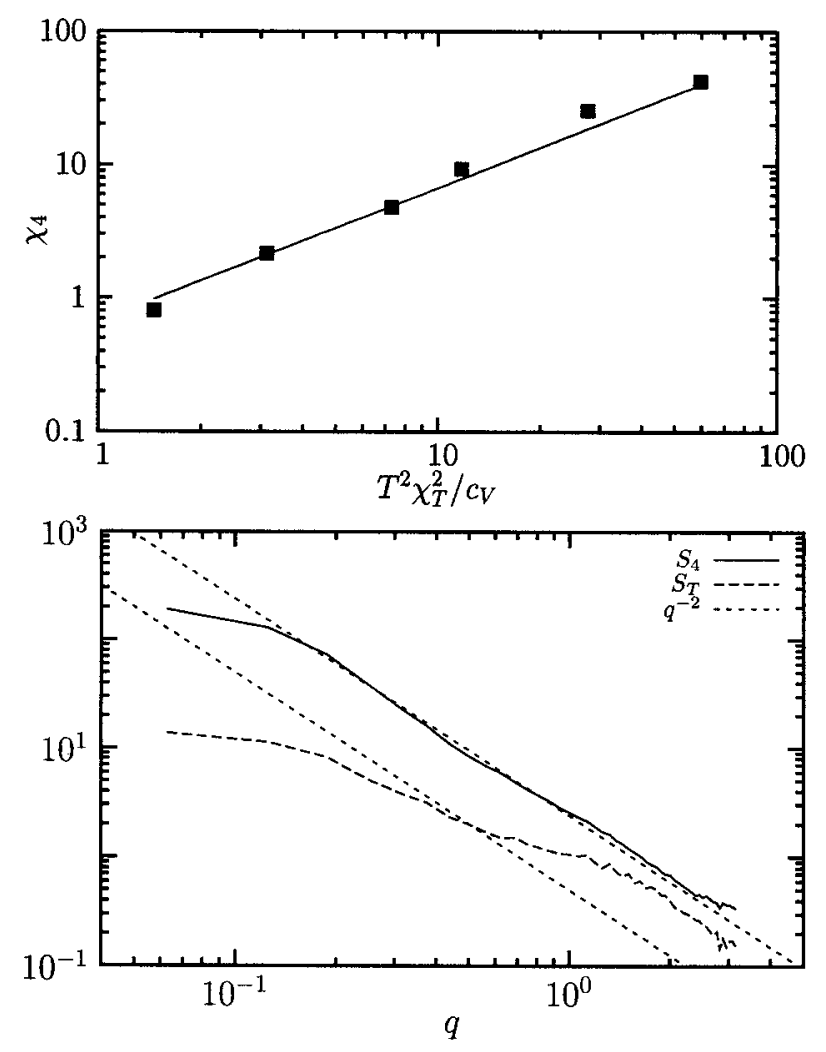

FIG. 8. Dynamic susceptibilities and spatial correlations in the two-spin facilitated FA model in two dimensions. Top: Comparison between the peak values of $\chi_{4}$ and $T^{2} \chi_{T}^{2} / c_{V}$ for different temperatures covering about 7 decades in relaxation time scales. The full line represents the proportionality between both quantities. Bottom: Dynamic structure factors $S_{4}(q, t)$ and $S_{T}(q, l)$ at time $t=\tau_{\alpha} \sim 10^{6}$ for $T=0.428$. Both functions behave as a constant at small $q$ but have different large $q$ behaviors since the $1 / q^{2}$ dashed line is consistent with $S_{4}$ only.

veals that the shapes of the dynamic structure factors $S_{4}$ and $S_{T}$ differ. While $S_{4} \sim 1 / q^{2}$ seems to hold at large wave vectors, $q \xi_{4} \gg 1$, we find a different behavior for $S_{T}$, namely, $S_{T} \sim 1 / q^{1.3}$. Note that this fit is not very satisfactory, revealing a more complex structure of this correlator, possibly related to the presence of two length scales in the model: the size of the superdefects and the typical distance separating them. We conclude that dynamic-dynamic and dynamicenergy correlations might contain slightly distinct physical information in cooperative KCMs. This is physically expected because an isolated defect, which represents a positive local fluctuation of the energy, cannot diffuse and relax the neighboring sites. Therefore the correspondence between energy fluctuations and dynamical fluctuations is not one to one as in, e.g., the one-spin FA model. By this argument one can predict that $S_{T}(r, t)<S_{4}(r, t)$ at small $r$ and therefore a faster initial decay of $S_{T}(r, t)$ with $r$. In Fourier space, this means a slower large $q$ decay of $S_{T}(q, t)$ than that of $S_{4}(q, t)$, as observed in Fig. 8.

Nevertheless, a dynamic correlation length scale can be defined from both $S_{4}(q, t)$ and $S_{T}(q, t)$ as the inverse of the wave vector above which structure factors start to decay. The data shown in Fig. 8 clearly indicate that these two length scales are very close. A possible interpretation is that despite their complex structure, superdefects remain associated with some positive energy fluctuations, so that the length scale 
extracted from three- and four-point functions could indeed be equivalent, as in the case of noncooperative models. A similar situation was encountered in our atomistic simulations in I.

\section{Remarks and open questions on ensemble and dynamics dependence and KCMs}

We have studied in the context of KCMs the dynamic susceptibility $\chi_{T}(t)$ and the associated three-point dynamicsenergy spatial correlations and their link with the more standard four-point susceptibility $\chi_{4}(t)$. Although the thermodynamic relations derived in I for supercooled liquids do not hold for kinetically constrained spin models (because energy is not dynamically conserved), they seem to be approximately valid.

The underlying reason is that in noncooperative KCMs the energy fluctuations that are important for the dynamics are effectively conserved because of the kinetic constraint. This is clearer in the example of the one-spin facilitated FA model where a facilitating spin can disappear only by annihilation with another facilitating spin. Similarly, a facilitating spin can be created only by branching from another facilitating spin. But these two processes happen very rarely (see Refs. 47, 58, and 59 for a detailed analysis and discussion of time scales). Therefore, the main relaxation mechanism is diffusion of the facilitating regions (energy fluctuations) which are conserved in an effective way, as assumed in the independent defect approximation.

Comparing our results for KCMs to the general theoretical considerations of I opens interesting issues related to the applicability of KCMs to supercooled liquids. Since dynamical fluctuations strongly depend on statistical ensembles and microscopic dynamics, this immediately raises important questions:

- For which ensemble are the dynamical fluctuations of real liquids supposed to be described by KCMs?

- What type of liquid dynamics should one choose to compare real dynamical fluctuations to the prediction of KCMs?

These questions are clearly related to the coarse-graining procedure that is often invoked, ${ }^{51,54,70}$ but never truly performed, to map real liquids to KCMs. Were this procedure known, the answer to the previous questions would be clear. Unfortunately, this formidable task has not yet been accomplished. On the other hand, our results show that this issue is important if one wants to compare KCM predictions for dynamic susceptibilities $\chi_{4}$ and $\chi_{T}$ to experimental and numerical results on realistic models.

For kinetically constrained spin models, the answer to the first of the above questions seems fairly easy even without the coarse-graining procedure. Only in the most general ensemble where all conserved quantities fluctuate does one have $\lim _{q \rightarrow 0} S_{4}(q, t)=S_{4}(0, t)$. Since this equality holds in $\mathrm{KCM}$, we conclude that KCMs should apply to real liquids in the most general statistical ensemble, i.e., NPT for most practical purposes.
The second question is instead much more subtle. From a general point of view since there are no conserved quantities in spin models, KCMs could be thought as representative of a dynamics without conserved quantities. Of course all physical dynamics should at least conserve density. However, if one considers Brownian dynamics for supercooled liquids for which temperature is the relevant control parameter, while density plays a minor role (see Sec. II E 3 in I), it might be reasonable to expect that density fluctuations do not couple strongly to dynamical fluctuations. One is then tempted to conclude that KCMs are models of real liquids with Brownian or stochastic dynamics.

However, this tentative answer is contradicted by several facts. First, real supercooled liquids obviously evolve with Newtonian dynamics. Second, we just discovered that the inequality (31) provides a good approximation to $\chi_{4}(t)$ for KCMs. A similar result holds for liquids with Newtonian dynamics in the NPT ensemble (see I) but not for liquids with stochastic dynamics. ${ }^{71}$

Taking the opposite view that KCMs represent, for some unclear reason, liquids with Newtonian dynamics is also unsatisfactory because the saturation of the inequality (31) in KCMs is principally due to the behavior of the specific heat that decreases exponentially fast as temperature decreases. But a very small specific heat is incompatible with experimental measurements of the thermodynamics of supercooled liquids. ${ }^{72}$ Correcting for this fact as in Ref. 73 then leads to poor estimates of $\chi_{4}(t)$ via dynamic response functions, in disagreement with atomistic simulations. ${ }^{16,19}$

The case of kinetically constrained lattice gases is less problematic if taken as models of glass/jamming transition in hard sphere systems, rather than molecular liquids. In this case, the only conserved quantity that matters is the density and therefore there are no ambiguities since density is conserved both in kinetic lattice gases and in real systems.

KCMs provide a natural mechanism explaining correlations between energy fluctuations and dynamic heterogeneity. However, in order to compare even qualitative predictions of KCMs with experimental or numerical results for dynamical fluctuations, one has to understand clearly in what ensemble and for what dynamics KCM predictions hold. This certainly highlights the importance of a microscopic derivation and more detailed justification of KCMs.

\section{NUMERICAL RESULTS FOR TWO MOLECULAR GLASS FORMERS}

\section{A. Models and technical details}

In this section we report our numerical calculations of the dynamic susceptibility $\chi_{T}(t)$ in two molecular glass formers which have been extensively studied in numerical simulations: a binary LJ mixture, ${ }^{74}$ considered as a simple model system for fragile supercooled liquids, ${ }^{6}$ and the van Beest, Kramer, and van Santen (BKS) model, which is a simple description of the strong glass-former silica. ${ }^{61,75}$ For both models we have investigated the behavior of the dynamical fluctuations performing microcanonical simulations at constant energy $E$, number of particles $N$, and volume $V$, by solving Newton's equations of motion. ${ }^{76}$ For the LJ system 
we have also simulated two types of stochastic dynamics, namely, Brownian and Monte Carlo dynamics. ${ }^{76}$

We follow the dynamical behavior of the molecular liquids through the self-intermediate scattering function

$$
F_{s}(\mathbf{k}, t)=\left\langle\frac{1}{N_{\alpha}} \sum_{j=1}^{N_{\alpha}} e^{i \mathbf{k} \cdot\left[\mathbf{r}_{j}(t)-\mathbf{r}_{j}(0)\right]}\right\rangle,
$$

where the sum in Eq. (49) runs over one of the species of the considered liquid ( $A$ or $B$ in the $\mathrm{LJ}, \mathrm{Si}$ or $\mathrm{O}$ for silica). We denote by $f_{s}(\mathbf{k}, t)$ the real part of the instantaneous value of this quantity, so that we have $F_{s}(\mathbf{k}, t)=\left\langle f_{s}(\mathbf{k}, t)\right\rangle$.

As usual, the four-point susceptibility $\chi_{4}(t)$ quantifies the strength of the spontaneous fluctuations around the average dynamics by their variance

$$
\chi_{4}(t)=N_{\alpha}\left[\left\langle f_{s}^{2}(\mathbf{k}, t)\right\rangle-F_{s}^{2}(\mathbf{k}, t)\right] .
$$

In principle, $\chi_{4}(t)$ in Eq. (50) retains a dependence on the scattering vector $\mathbf{k}$. Since the system is isotropic, we circularly average (49) and (50) over wave vectors of fixed modulus, and we mainly consider results for $|\mathbf{k}|=7.21$ for the LJ system, and $|\mathbf{k}|=1.7 \AA^{-1}$ for the BKS. These values, respectively, represent the typical distance between $A$ particles, and the size of the $\mathrm{SiO}_{4}$ tetrahedra. Finally, we use finite difference to evaluate the temperature derivatives involved in

$$
\chi_{T}(t)=\frac{\partial}{\partial T} F_{s}(\mathbf{k}, t) .
$$

We have given an extensive account of the models, numerical details, and parameters used in I. Therefore, we refer readers interested in the technical details concerning the simulations to I. ${ }^{16}$ Note also that we will neglect, as justified in I, the role density fluctuations on dynamical correlations. Therefore we will focus on $\chi_{4}^{N V T}$ (instead of $\chi_{4}^{N P T}$ ) and $\chi_{T}$ obtained deriving with respect to temperature at fixed volume (and not fixed pressure).

\section{B. Time dependence of dynamic susceptibilities \\ 1. Time behavior of $\chi_{T}(t)$}

Our results for the dynamic susceptibilities $\chi_{T}(t)$ are presented in Fig. 9 for both the LJ and BKS models. For a given temperature, the qualitative time dependence of $\chi_{T}(t)$ observed in Fig. 9 resembles the one already reported for $\chi_{4}(t)$ : $\chi_{T}(t)$ presents a peak for a time scale close to $\tau_{\alpha}$. This is very natural since by definition $\chi_{T}(t=0)=\chi_{T}(t \rightarrow \infty)=0$, and it is for times $t \approx \tau_{\alpha}$ that the dynamics is most sensitive to temperature changes. We have shown the quantity $\left|\chi_{T}(t)\right|$ in these figures, as $\chi_{T}(t)$ is obviously a negative quantity: raising the temperature makes the dynamics faster, and hence two-time correlators smaller, so that $\partial F_{s} / \partial T<0$.

More quantitatively, we expect the two-time-scale relaxation of the averaged dynamics to lead to a complex time behavior of $\chi_{T}(t)$, similar to that predicted for $\chi_{4}(t) .{ }^{14}$ Within MCT, we expect (see Sec. II) two distinct power laws, $\chi_{T}$ $\sim t^{a}$ followed by $\chi_{T} \sim t^{b}$, to describe the approach to the maximum of $\chi_{T}$, the exponents $a$ and $b$ being already constrained to the values they take when fitting the averaged dynamics using MCT. From the study of KCMs only the
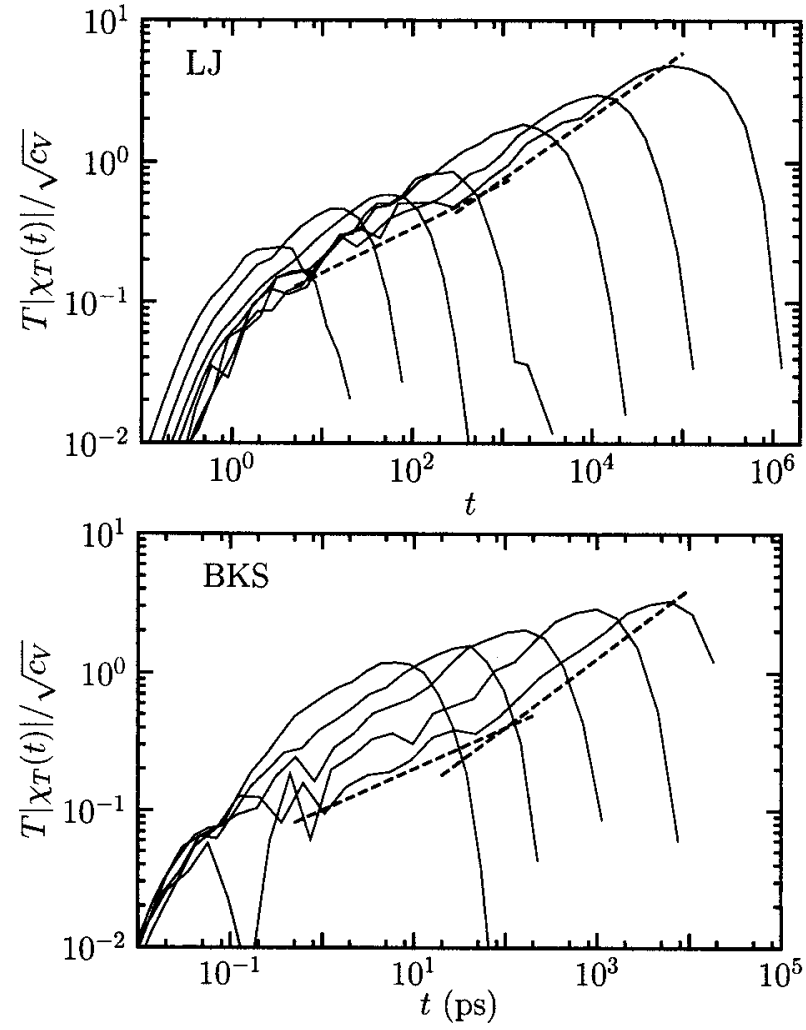

FIG. 9. Normalized $\chi_{T}$ as a function of time for various temperatures in a binary Lennard-Jones mixture (top) and the BKS model for silica (bottom), obtained from molecular dynamics numerical simulations. LJ: $T=2.0,1.0$, $0.75,0.6,0.5,0.465$, and 0.432 from left to right. BKS $T=6000,4650,4000$, $3550,3200,3000$, and $2730 \mathrm{~K}$ from left to right. We have taken the absolute value since $\chi_{T}$ is a negative quantity. Power law fits of the time dependence are discussed in detail in Sec. IV. The values of the exponents at short and long times are 0.32 and 0.45 in LJ and 0.3 and 0.5 in BKS.

approach to the peak can be predicted since the short-time dynamics contains no clear relaxation towards a plateau due to the coarse-grained nature of the models. ${ }^{14,48,63}$ Again, a power law approach to the peak is expected.

In Fig. 9 we compare our numerical results for $\chi_{T}(t)$ to power law behaviors shown as dashed lines. On the restricted time window of the simulations there is obviously some freedom in the fitting procedure so the exponents we report should be considered as an empirical quantitative description of the true time dependence of these functions. As discussed already in the case of $\chi_{4}(t),{ }^{14}$ corrections to the asymptotic scaling laws derived by theoretical approaches should be expected in the reduced time regime of the molecular simulations. In the LJ system we find that the time behavior of $\chi_{T}(t)$ can be described by the exponents $a \approx 0.32$ and $b \approx 0.45$ with the tendency that these exponents very slowly decrease when $T$ decreases. For the BKS system we find a similar quality of the fits with $a \approx 0.3$ and $b \approx 0.5$ with no systematic dependence in temperature.

The values of these exponents compare reasonably well with the MCT predictions obtained above. For the LJ system, the von Schweidler exponent is estimated to be $b \approx 0.51$ from fitting the averaged dynamics in the $\beta$-relaxation regime, ${ }^{74}$ while direct computations predict $b=0.62$. $^{77}$ Both values are close to our finding, $b \approx 0.45$, although they both slightly overestimate it. The exponent $a$ describing the dynamics in 
TABLE I. Summary of the different results for exponents $\theta, a$, and $b$, describing the peak amplitude and the time dependence of $T\left|\chi_{T}\right| / \sqrt{c_{V}}$ and $\mathrm{X}_{4}^{N V E}$ (see text). NA, not applicable.

\begin{tabular}{lcccccc}
\hline \hline Observable & LJ & BKS & MCT (LJ) & MCT (BKS) & KCM (1FA) & KCM (East) \\
\hline$\theta\left(\chi_{T}\right)$ & 0.33 & 0.14 & 0.43 & 0.43 & 0.25 & $\propto T$ \\
$\theta\left(\chi_{4}^{N V E}\right)$ & 0.39 & 0.18 & 0.43 & 0.43 & $0.5^{\mathrm{a}}$ & $\propto T$ \\
$a\left(\chi_{T}\right)$ & 0.32 & 0.3 & $0.29-0.32$ & 0.32 & NA & NA \\
$a\left(\chi_{4}^{N V E}\right)$ & $0.37^{\mathrm{b}}$ & $\mathrm{NA}$ & $0.29-0.32$ & 0.32 & $\mathrm{NA}$ & NA \\
$b\left(\chi_{T}\right)$ & 0.45 & 0.5 & $0.51-0.62$ & 0.62 & 1 & $\beta(T) \propto T$ \\
$b\left(\chi_{4}^{N V E}\right)$ & 0.7 & $0.65-0.85$ & $0.51-0.62$ & 0.62 & $2^{\mathrm{a}}$ & $\beta(T) \propto T$ \\
\hline \hline
\end{tabular}

${ }^{2}$ Ambiguous-do KCMs describe $\chi_{4}^{N V T}$ Newtonian or $\chi_{4}^{N V T}$ Brownian $\left(=\mathrm{X}_{4}^{N V E}\right)$ ?

${ }^{\mathrm{b}}$ Obtained from MC dynamics.

the early $\beta$ regime was not directly fitted, but using the known relations between MCT exponents their values are predicted to be $a=0.29$ (for $b=0.51$ ) and $a=0.32$ (for $b$ $=0.62$ ). This is again consistent with our finding for $\chi_{T}(t)$, $a \approx 0.32$, in this time regime. From the point of view of MCT, we suggest that focusing on $\chi_{T}$ is a more powerful way to directly measure the exponent $a$ (this might be interesting from an experimental point of view as well). Finally for BKS, fitting of the average dynamics provides the value $b=0.62$, from which $a=0.32$ is deduced from known MCT relations. ${ }^{61}$ These two values again compare relatively well with the time behavior found for $\chi_{T}(t)$, namely, $a \approx 0.3$ and $b \approx 0.5$.

Applying results from $\mathrm{KCMs}$ to real liquids, one would predict the time dependence of $\chi_{T}(t)$ when approaching the peak to be $\chi_{T}(t) \sim t$ for an Arrhenius liquid modeled by the one-spin facilitated model in three dimensions, while $\chi_{T}(t)$ $\sim t^{b(T)}$ is predicted for fragile liquids modeled by the East model. Our numerical results for BKS silica are not consistent with the FA model predictions and are, quite unexpectedly, more compatible with the smaller exponents observed in the fragile East model reported in Sec. III B. The small $b(T)$ exponents of the East model compare, however, well with the behavior of $\chi_{T}(t)$ found in the LJ system. In particular, the fact that $b(T)$ decreases with decreasing $T$ is correctly predicted by fragile KCMs, as opposed to the constant $b$ predicted by MCT. For a summary of these results, see Table I.

\section{Comparison between $\chi_{4}(t)$ and $\chi_{T}(t)$}

It is interesting to compare the exponents found numerically for $\chi_{T}(t)$ to the ones of $\chi_{4}(t)$ measured in the $N V E$ ensemble for Newtonian dynamics since theory predicts some relations between them. The latter exponents were already studied in Ref. 14 for the LJ. Numerically no power law behavior $\chi_{4}(t) \sim t^{a}$ is found in the short-time behavior of $\chi_{4}(t)$ in the Newtonian dynamics of both the LJ and BKS systems. This is due to the fact that thermal vibrations strongly affect the short-time dynamics of these liquids. Two power law regimes are, however, clearly observed in the stochastic simulations where phonons are either overdamped (Brownian dynamics) or absent (Monte Carlo dynamics). Our Monte Carlo results for $\chi_{4}(t)$ in the LJ are presented in Fig. 10 (top) where we have fitted the early and late $\beta$ regimes with two power laws with exponents $a \approx 0.37$ and $b$ $\approx 0.7$, respectively. For the BKS we performed Newtonian dynamics simulations only. Hence, we only have results on the exponent $b$ from $\chi_{4}$ measurements, which is found to increase from 0.65 to 0.85 upon lowering the temperature: this is an opposite behavior compared to the $\mathrm{LJ}$ where $b$ decreases. This might suggest a different temperature behavior of $b$ in strong and fragile liquids. This trend is partly captured by KCMs.

MCT predicts that $\chi_{T}(t)$ and $\chi_{4}^{N V E}(t)$ have the same critical scaling. KCM predictions are ambiguous so we follow the numerical results obtained in Sec. III, i.e., $\chi_{4}(t) \sim \chi_{T}^{2}(t)$. In both LJ and BKS systems, the exponent $a$ is the same for both susceptibilities, as predicted by MCT. The results for $b$
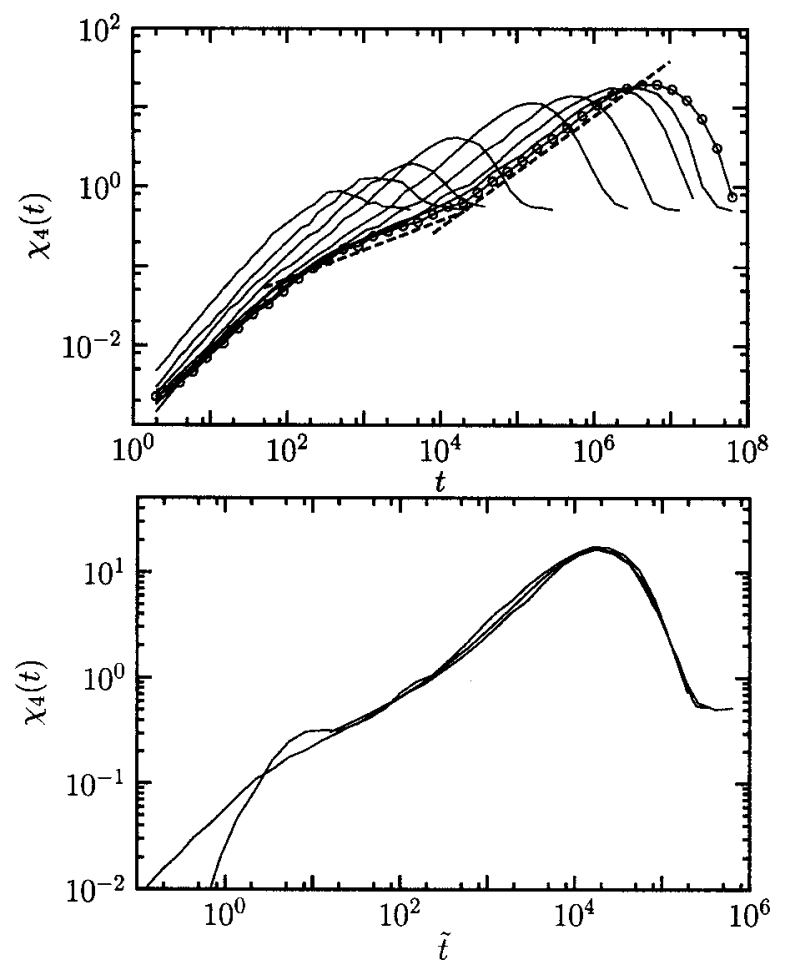

FIG. 10. Top: Four-point susceptibility $\chi_{4}(t)$ in the binary LJ mixture with Monte Carlo dynamics for $T=2.0,1.0,0.75,0.6,0.5,0.47,0.45$, and 0.43 (from left to right), the lowest temperature being highlighted with open circles. Power laws $\chi_{4} \sim t^{0.37}$ and $\chi_{4} \sim t^{0.7}$ are indicated with dashed lines in the early and late $\beta$ regimes, respectively. Bottom: $\chi_{4}(t)$ is shown for $T$ $=0.45$ for $N V E$ Newtonian, Brownian, and Monte Carlo dynamics as a function of a rescaled time chosen so that all $\chi_{4}$ 's overlap near the alpha relation. We chose $\tilde{t}=t$ for $N V E$ Newtonian dynamics, $\tilde{t}=t / 24$ for Brownian dynamics, and $\tilde{t}=t / 100$ for Monte Carlo dynamics. No rescaling of the vertical axis is performed. The agreement between the three types of dynamics is remarkable. 

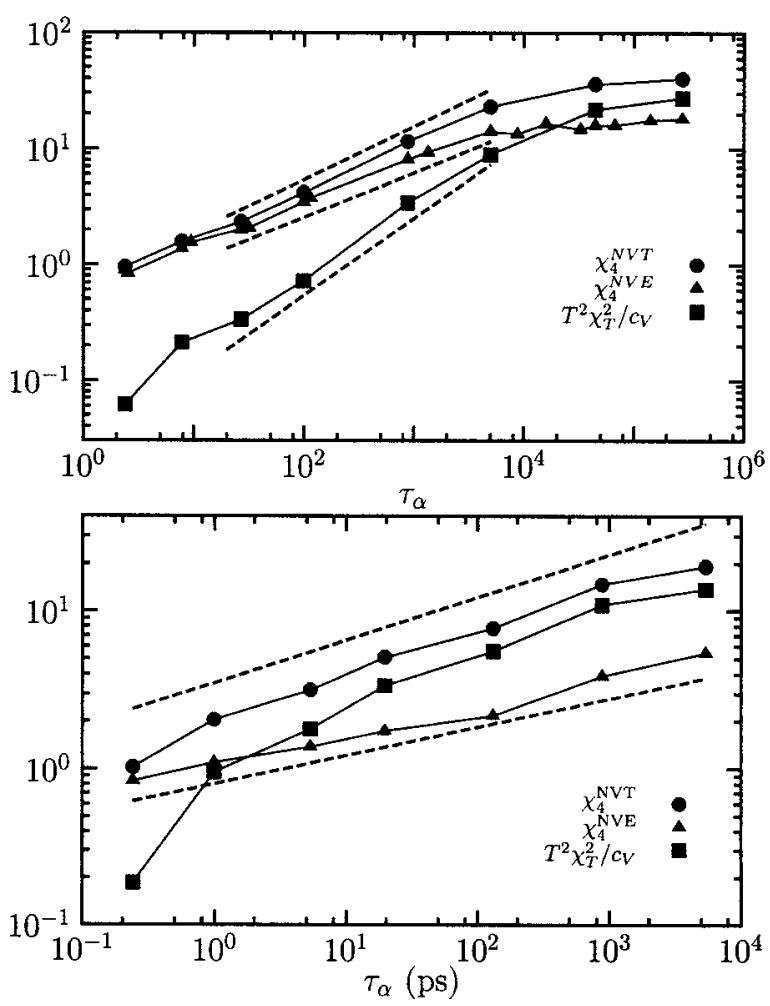

FIG. 11. Various susceptibilities in the binary LJ mixture obtained from the A particles dynamics (top) and the BKS model for silica from the Si ion dynamics (bottom). Dashed lines indicate power law behavior with exponents $0.46,0.39$, and 0.67 (from top to bottom in the LJ system) and 0.27 and 0.18 (from top to bottom in the BKS model). In all cases, $T^{2} \chi_{T}^{2} / c_{V}$ is smaller than $\chi_{4}^{N V E}$ at high temperature but increases faster and becomes eventually the dominant contribution to $\chi_{4}^{N V T}$ in the relevant low temperature glassy regime.

are more difficult to interpret: although $b$ for $\chi_{4}$ is systematically larger than for $\chi_{T}$, the ratio between the two exponents is not 2 either, so that neither MCT nor KCM approaches really describe this aspect of our numerical results. For a summary of these results, see Table I.

What comes nicely out of the simulations, however, is the fact, predicted on general grounds in I and within MCT above, that NVE Newtonian, Brownian, and Monte Carlo dynamics display similar time dependencies for the dynamic susceptibility $\chi_{4}(t)$. This is strikingly illustrated in Fig. 10 (bottom) which shows $\chi_{4}(t)$ at a single temperature, $T$ $=0.45$. The results for the three dynamics almost perfectly overlap for time scales larger than the plateau regime in $F_{s}(\mathbf{k}, t)$.

\section{Peak amplitude of dynamic fluctuations}

We now focus on the amplitude of the peak observed in the various susceptibilities. In Fig. 11, we present our numerical results for $\chi_{4}^{N V E}, T^{2} \chi_{T}^{2} / c_{V}$, and their sum $\chi_{4}^{N V T}$ obtained from the Newtonian dynamics of both the LJ and BKS models. When temperature decreases, all peaks shift to larger times and track the $\alpha$ relaxation. Simultaneously, their height increases, revealing increasingly larger dynamic fluctuations as the glass transition is approached.

The main observation from the data displayed in Fig. 11, already made in Ref. 19 and in I, is that in both LJ and BKS systems the term $T^{2} \chi_{T}^{2} / c_{V}$ while being small, $\sim O\left(10^{-1}\right)$, above the onset temperature of slow dynamics, grows much faster than $\chi_{4}^{N V E}$ when the glassy regime is entered. As a consequence, there exists a temperature below which the temperature derivative contribution to the four-point susceptibility $\chi_{4}^{N V T}$ dominates over that of $\chi_{4}^{N V E}$. This crossover is located at $T \approx 0.45$ in the LJ system and $T \approx 4500 \mathrm{~K}$ for BKS silica. Remarkably, the conclusion that $T^{2} \chi_{T}^{2} / c_{V}$ becomes larger than $\chi_{4}^{N V E}$ at low temperatures holds for both strong and fragile glass formers. Experimental and theoretical consequences of this observation were discussed in Refs. 16 and 19.

Following Ref. 47 we have chosen to present the evolution of the amplitude of the dynamic susceptibilities as a function of $\tau_{\alpha}$ rather than $T$ because it is in this representation that dynamic scaling might emerge. For the LJ system we find that all susceptibilities can be described by power laws, $\chi \sim \tau_{\alpha}^{\theta}$, in some intermediate, and therefore subjectively defined, temperature regime with the following exponents: $\theta \approx 0.39$ for $\chi_{4}^{N V E}, \theta \approx 0.46$ for $\chi_{4}^{N V T}$, and $\theta \approx 0.67$ for $T^{2} \chi_{T}^{2} / c_{V}$. For the BKS model, we find that $\theta \approx 0.27$ for both $\chi_{4}^{N V T}$ and, in a more restricted time window, $T^{2} \chi_{T}^{2} / c_{V}$ while we find that $\theta \approx 0.18$ for $\chi_{4}^{N V E}$.

The theoretical considerations given above show that these exponents should be related, within MCT, to the exponent $\gamma$ describing the divergence of $\tau_{\alpha}$ close to $T_{c}$. The prediction is that $\theta=1 / \gamma$ for $\chi_{4}^{N V E}$ and $T \chi_{T} / \sqrt{c_{V}{ }^{92}}$ while $\theta$ $=2 / \gamma$ for $\chi_{4}^{N V T}$ and $T^{2} \chi_{T}^{2} / c_{V}$. Fitting of the relaxation times has shown that $\gamma \approx 2.35$ for both LJ and BKS systems, so the exponents $1 / \gamma=0.426$ and $2 / \gamma=0.851$ should be observed in Fig. 11. The exponent for $\chi_{4}^{N V E}$ is reasonably well described by MCT predictions in the LJ system, an agreement already reported in Refs. 34 and 71 (see Table I). The agreement deteriorates somewhat for $T \chi_{T} / \sqrt{c_{V}}$. The MCT predictions fail, however, strongly in the BKS system, for which the value of 0.18 is found instead of the expected 0.426 for $\chi_{4}^{N V E}$ and $\chi_{T}$, although in a temperature regime where Arrhenius behavior is already observed. No clear power law can be seen in the mode-coupling regime seen in Ref. 61. In principle, the behavior of $\chi_{T}(t)$ is completely tied to the one of the average two-time correlators already studied in Ref. 51, but $\chi_{T}(t)$ provides a more detailed analysis of the dynamics with no fitting procedure required. Therefore the failure of MCT to capture the behavior of $\chi_{T}(t)$ suggests that MCT, despite the claims of Ref. 61, does not satisfactorily describe the dynamical behavior of this strong glass former.

Finally we find that $T^{2} \chi_{T}^{2} / c_{V}$ and $\chi_{4}^{N V T}$ behave somewhat differently in the temperature regime where power law fits are performed. This is not surprising. We have extensively discussed in I the fact that simulations are typically performed in the relatively high temperature regime where both terms contributing to $\chi_{4}^{N V T}$ are comparable. Since they are predicted to have different scaling behaviors, the intermediate value for the exponent $\theta$ reported for $\chi_{4}^{N V T}$ simply results from this crossover.

The power law regimes we have discussed do not describe the whole temperature range studied for the LJ system. For $T \leqq 0.47$ the growth of all dynamic susceptibilities with $\tau_{\alpha}$ becomes much slower, perhaps logarithmically slow, but 
we do not have a sufficient range of time scales in this low temperature regime to draw more quantitative conclusions. We have moreover checked that this saturation is not the finite size effect expected if fluctuations are computed in too small a system size ${ }^{67}$ see I. Interestingly, no such saturation can be observed in the BKS system. Therefore we do not know how to extrapolate the present numerical results towards the glass transition temperature, and compare our simulations to the result, reported in Ref. 19, that dynamic susceptibilities have typically the same value at $T_{g}$ for liquids with very different fragilities. We can simply state from our results that this fragility independence cannot hold at all temperatures since Fig. 11 clearly shows that dynamic susceptibilities grow at different rates in different systems. We are currently investigating this point in more detail. ${ }^{78}$

The saturation of the LJ dynamic susceptibilities observed at low $T$ seems consistent with the theoretical expectation, ${ }^{14,33,63,70,79-81}$ and the experimental confirmation $^{19,82-84}$ that dynamic fluctuations and length scales grow very slowly when $T$ is decreased towards $T_{g}$. From the fragile KCM perspective, one would, for instance, expect that $\chi_{4} \sim \tau_{\alpha}^{\theta(T)}$ with an exponent $\theta(T)$ which decreases linearly with $T,{ }^{63,70}$ while logarithmic growth, $\chi_{4}$ $\sim\left(\log \tau_{\alpha}\right)^{\psi}$, is predicted by activation based theories. ${ }^{33,79,81}$

\section{CONCLUSIONS AND PERSPECTIVES}

This paper describes the second part of our investigations of dynamical susceptibilities started in $\mathrm{I}^{16}$ In this second work we have illustrated the general conclusions of I by making explicit the predictions of MCT and KCMs concerning spontaneous dynamical fluctuations [encoded in $\chi_{4}(t)$ ] and induced one [given by $\chi_{x}(t)$ ]. These theories predict the detailed dependence of these two quantities both as a function of time and of temperature (or density). As discussed in I, special care must be devoted to the choice of statistical ensemble and microscopic dynamics, with the rather spectacular prediction of MCT that $\chi_{4}(t)$ should coincide (or at least display the same scaling) for Newtonian dynamics in the $N V E$ ensemble and for Brownian dynamics in the $N V T$ ensemble, but differ from the result for Newtonian dynamics in the $N V T$ ensemble. The predictions coming from KCMs are much less clear about this particular point, since there is some intrinsic ambiguity about which ensemble and which dynamics these models are supposed to describe.

We have compared these predictions with numerical simulations of models of supercooled liquids. Overall, as shown in Table I, MCT fares reasonably well at accounting for the detailed shape of $\chi_{4}(t)$ and $\chi_{T}(t)$ of the Lennard-Jones system in a restricted temperature region where MCT can be applied. As for the values of the exponents, our aim was to present a rather qualitative comparison focusing more on compatibility than on precise tests, which are beyond the scope of this work, and probably of MCT as well. Quite remarkably, the exponents used to fit these higher order correlations are indeed compatible with those measured on twopoint correlation functions, with quantitative variations that can perhaps be attributed to preasymptotic effects. Furthermore, the predicted ensemble dependence of these quantities is very clearly highlighted by our numerical results. We have also shown that the wave vector dependence of $\chi_{4}(t)$ can be qualitatively accounted for within MCT. On the other hand, the features of the dynamical susceptibility of the BKS model for the strong silica glass are not quantitatively well explained by MCT. Similarly KCMs fail to describe quantitatively the results obtained in the BKS model, but the systematic temperature dependence of the exponents describing $\chi_{4}(t)$ appears somewhat natural from this perspective.

Among open problems, we should primarily emphasize the major problem of extending MCT to allow for activated events. A detailed prediction of $\chi_{4}(t)$ and of the geometry and exponents of dynamically correlated regions in the deeply supercooled region would be important to compare with future experiments (see Refs. 14, 31, and 85 for preliminary elements in that direction). The generalization of these predictions to the aging regime would also certainly be relevant to analyze the cooperative dynamics of deeply quenched glasses.

\section{ACKNOWLEDGMENTS}

The authors thank L. Cipelletti, S. Franz, F. Ladieu, A. Lefèvre, D. L'Hôte, G. Szamel, and G. Tarjus for discussions. Two of the authors (D.R.R. and K.M.) acknowledge support from the NSF (NSF CHE-0134969). Another author (G.B.) is partially supported by EU Contract No. HPRN-CT2002-00307 (DYGLAGE-MEM). Another author (K.M.) would like to thank J. D. Eaves for his help on the development of efficient numerical codes.

\section{APPENDIX A: GENERAL DISCUSSION AND RESULTS}

Much intuition concerning dynamic heterogeneity has been gleaned from the study of mean-field spin glasses. In particular, Franz and Parisi first pointed out that a quantity analogous to $\chi_{4}(t)$, which can be computed exactly in meanfield $p$-spin models, should show nontrivial features, ${ }^{11}$ which prompted the study of dynamic fluctuations in simulations of atomistic glass-forming liquids. ${ }^{12}$ The growth of a dynamic susceptibility in this model was properly interpreted in terms of a growing dynamical length scale, which diverges at $T_{c}$. The same scenario, complete with a temporal behavior of $\chi_{4}(t)$ identical to that in the $p$-spin models, exists in meanfield models that have no underlying thermodynamic critical point. ${ }^{56,86}$ It should also be noted that this scenario is perhaps more general than appreciated, since it appears to also exist in models on compact lattices with no quenched disorder and short-ranged interactions, at least in the limit of large dimensionality ${ }^{87}$ and models with long-ranged, Kac-type interactions. ${ }^{88,89}$

Applying the above diagrammatic analysis to $p$-spin models for which no conserved quantities exist, one finds, in agreement with $\mathrm{BB}$, that $\chi_{4}(t)$ is determined by ladder diagrams only. Hence, its critical behavior has to be the same as the dynamical response $\chi_{T}(t)$ and is given by Eq. (11). Similarly the susceptibility $\chi_{\mathrm{FP}}(t)$ introduced by Franz and Parisi is found to follow the same scaling behavior. As discussed below, Franz and Parisi ${ }^{11}$ study the quantity $\chi_{\mathrm{FP}}(t)$ $=d C(t) / d \epsilon$, where $C(t)=\left\langle s_{i}(t) s_{i}(0)\right\rangle$ and $\epsilon$ is an infinitesimal 


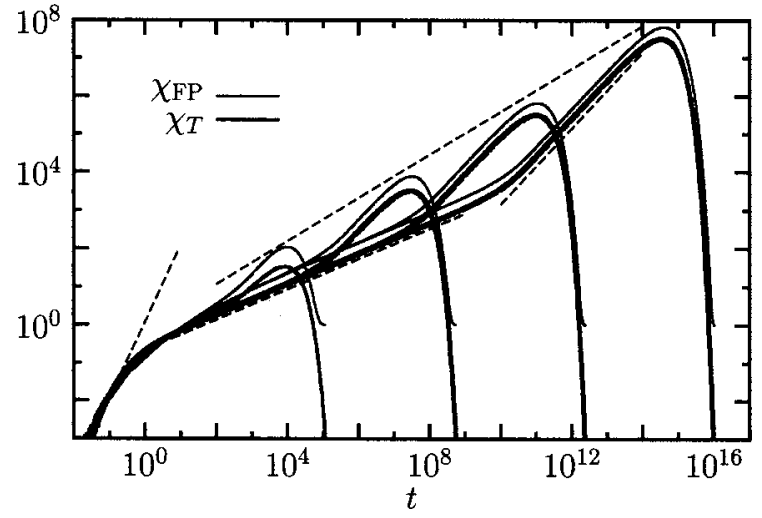

FIG. 12. Time dependence of the dynamic susceptibilities $\chi_{T}(t)$ (thick lines) and $\chi_{\mathrm{FP}}(t)$ (thin lines) in the $p=3$ mean-field $p$-spin model for temperatures approaching $T_{c}$ from above. Note the wide range of time scales covered in this graph. From left to right, $\left(T-T_{c}\right) / T_{c}=10^{-2}, 10^{-4}, 10^{-6}, 10^{-8}$. The asymptotic power law regimes are shown as dashed lines. The susceptibilities grow as $t^{2}, t^{a}$, and $t^{b}$ in the microscopic, early, and late beta regimes, while the height of the maxima scales as $\chi^{*} \sim \tau^{1 / \gamma}$. For $p=3$, one has $a$ $=0.395, b=1$, and $\gamma=1.765$.

field coupling the system's configuration at time $t$ to its initial state at time 0 . Using linear response theory they argue that $d C(t) / d \epsilon$ and $\chi_{4}(t)$ are equal. We find instead that $d C(t) / d \epsilon$ is equal to the sum of $\chi_{4}(t)$ and another nonvanishing contribution. However, $\quad d C(t) / d \epsilon=\chi_{\mathrm{FP}}(t)$ $=N^{-1} \sum_{i j} \int_{0}^{t} d t^{\prime}\left\langle s_{i}(t) s_{i}(0) s_{j}\left(t^{\prime}\right) \hat{s}_{j}\left(t^{\prime+}\right)\right\rangle$, where $\hat{s}_{i}(t)$ are the response field. Hence, it is given by ladder diagrams similar to the ones contributing to $\chi_{4}(t)$. Thus we expect that $\chi_{\mathrm{FP}}(t)$ and $\chi_{4}(t)$ behave similarly close to the critical point.

In the following, we shall present a careful numerical comparison between the dynamic susceptibility $\chi_{\mathrm{FP}}(t)$ and $\chi_{T}(t)$ integrating the integrodifferential equations derived in Ref. 11 for $p$-spin models. This comparison decisively confirms the previous analytical results. A much smaller time window was studied in Ref. 34, and it was not clear that asymptotic regimes had been observed.

One technical difficulty is that it is numerically difficult to calculate $\chi_{\mathrm{FP}}(t)$ very close to $T_{c}$. Here, we modify the method developed by Kim and Latz for the aging $p$-spin model $^{90}$ to accurately integrate the equations on $\chi_{\mathrm{FP}}(t)$ derived in Ref. 11 much closer to $T_{c}$ than has been reported in previous work. The dynamical equations are presented in Appendix B, while the details of the methodology are outlined in Appendix C. In the $p$-spin case, one can use an alternative way to compute $\chi_{\mathrm{FP}}(t)$ based on power counting in $N^{-1}$, the inverse number of spins. This provides a complementary way to show that dynamical fluctuations are indeed given by ladder diagrams.

Let us now present our numerical results. In Fig. 12, we show a comparison of $\chi_{\mathrm{FP}}(t)$ and $\chi_{T}(t)$ for various temperatures approaching $T_{c}$ from above. Clearly, $\chi_{T}(t)$ is remarkably similar to $\chi_{\mathrm{FP}}(t)$ in this regime, exhibiting a welldefined regime at short times that grows as a power law with the critical mode-coupling exponent $a=0.395$, and a welldefined power law at later times that grows with the von Schweidler exponent $b=1$. Note also that the height of the peak scales as $\tau^{1 / \gamma}$ (where $\tau$ is the relaxation time) for both functions, as predicted. When the transition temperature is approached from the nonergodic phase, only the first regime of slow growth with the exponent $a$ can be observed (not shown). These results represent a useful benchmark for the comparison with real liquids. Indeed, as presented in Fig. 10, $\chi_{4}(t)$ for Monte Carlo dynamics in a binary Lennard-Jones mixture (where vibrational modes that may obscure the exponent $a$ are absent) shows features strikingly similar to those of the $p$-spin model, complete with reasonably defined regimes showing both $a$ and $b$ exponents close to $T_{c}$.

\section{APPENDIX B: EXACT DYNAMICAL EQUATIONS}

Following Franz and Parisi, ${ }^{11}$ we consider the dynamic of a perturbed $p=3$ spherical $p$-spin model evolving with the Hamiltonian $H_{\text {tot }}(S)=H(S)-\epsilon \hat{C}\left(S, S_{0}\right)$, where $S_{t}$ is the spin state at time $t, \hat{C}\left(S, S^{\prime}\right) \equiv N^{-1} \sum_{i} S_{i} S_{i}^{\prime}$ is the overlap function, and $H(S)=\sum_{i<j<k} J_{i j k} S_{i} S_{j} S_{k}$ is the unperturbed $p$-spin Hamiltonian. The Franz-Parisi susceptibility is defined as the linear response of the two-point correlation function evaluated in the presence of the perturbation, $C_{\epsilon}(t, 0) \equiv\left\langle\hat{C}\left(S_{t}, S_{0}\right)\right\rangle_{\epsilon}$, as

$$
\chi_{\mathrm{FP}}(t)=\frac{\partial C_{\epsilon}(t, 0)}{\partial \epsilon} .
$$

The equations of motion for $C_{\epsilon}\left(t, t^{\prime}\right)$ and the associated response function $G_{\epsilon}\left(t, t^{\prime}\right)$ are derived using a standard Martin-Siggia-Rose (MSR) approach. ${ }^{11,40}$

$$
\begin{aligned}
\frac{\partial C_{\epsilon}\left(t, t^{\prime}\right)}{\partial t}= & -\mu(t) C_{\epsilon}\left(t, t^{\prime}\right) \\
& +\int_{0}^{t} d s f^{\prime \prime}\left(C_{\epsilon}(t, s)\right) G_{\epsilon}(t, s) C_{\epsilon}\left(s, t^{\prime}\right) \\
& +\int_{0}^{t^{\prime}} d s f^{\prime}\left(C_{\epsilon}(t, s)\right) G_{\epsilon}\left(t^{\prime}, s\right) \\
& +\beta f^{\prime}\left(C_{\epsilon}(t, 0)\right) C_{\epsilon}\left(t^{\prime}, 0\right)+\epsilon C_{\epsilon}\left(t^{\prime}, 0\right), \\
\frac{\partial G_{\epsilon}\left(t, t^{\prime}\right)}{\partial t}= & -\mu(t) G_{\epsilon}\left(t, t^{\prime}\right) \\
& +\int_{t^{\prime}}^{t} d s f^{\prime \prime}\left(C_{\epsilon}(t, s)\right) G_{\epsilon}(t, s) G_{\epsilon}\left(s, t^{\prime}\right),
\end{aligned}
$$

with the damping coefficient

$$
\begin{aligned}
\mu(t)= & T+\epsilon C_{\epsilon}(t, 0)+\beta f^{\prime}\left(C_{\epsilon}(t, 0)\right) C_{\epsilon}(t, 0) \\
& +\int_{0}^{t} d s\left\{f^{\prime \prime}\left(C_{\epsilon}(t, s)\right) G_{\epsilon}(t, s) C_{\epsilon}(t, s)\right. \\
& \left.+f^{\prime}\left(C_{\epsilon}(t, s)\right) G_{\epsilon}(t, s)\right\}
\end{aligned}
$$

and $f(x)=x^{3} / 2$. We have numerically solved these equations using the method described below. In the limit of $\epsilon \rightarrow 0$, we 
retrieve the equation of motion for the stationary state

$$
\frac{\partial C(t)}{\partial t}=-T C(t)+\frac{1}{2} \int_{0}^{t} d s C^{2}(t-s) \frac{\partial C(s)}{\partial s},
$$

where $C(t)=C_{\epsilon=0}(t, 0)$.

The temperature derivative $\chi_{T}(t)=\partial C(t) / \partial T$ is evaluated by simple numerical differentiation of $C(t)$ with finely spaced temperature points.

\section{APPENDIX C: NUMERICAL ALGORITHM}

In the following, we elucidate the technical detail to solve Eq. (B2). This is a natural generalization of an efficient algorithm to solve equilibrium mode-coupling equation developed by Fuchs et $a l^{49}$ to nonstationary systems. The method given here can also be applied for the aging dynamics. $^{90}$

First, we shall introduce a new quantity $Q_{\epsilon}\left(t, t^{\prime}\right)$ by

$$
Q_{\epsilon}\left(t, t^{\prime}\right) \equiv 1-C_{\epsilon}\left(t, t^{\prime}\right)-\int_{t^{\prime}}^{t} d s G_{\epsilon}(t, s),
$$

where the subscript $\epsilon$ has been omitted for simplification. This function monitors the degree of violation of the fluctuation-dissipation theorem. With this new function, the MCT equation, Eq. (B2), can be rewritten as

$$
\begin{aligned}
\frac{\partial C_{\epsilon}\left(t, t^{\prime}\right)}{\partial t}= & -\mu^{\prime}(t) C_{\epsilon}\left(t, t^{\prime}\right)-\int_{t^{\prime}}^{t} d s\left[f^{\prime}(t, s) \frac{\partial C_{\epsilon}\left(s, t^{\prime}\right)}{\partial s}\right. \\
& \left.-f^{\prime \prime}(t, s) \frac{\partial Q_{\epsilon}(t, s)}{\partial s} C_{\epsilon}\left(s, t^{\prime}\right)\right]+P_{\epsilon}\left(t, t^{\prime}\right), \\
\frac{\partial Q_{\epsilon}\left(t, t^{\prime}\right)}{\partial t}= & -1+\mu^{\prime}(t)-\mu^{\prime}(t) Q_{\epsilon}\left(t, t^{\prime}\right) \\
& -\int_{t^{\prime}}^{t} d s\left[f^{\prime}(t, s) \frac{\partial Q_{\epsilon}\left(s, t^{\prime}\right)}{\partial s}+f^{\prime \prime}(t, s) \frac{\partial Q_{\epsilon}(t, s)}{\partial s}\right. \\
& \left.\times\left\{1-Q_{\epsilon}\left(s, t^{\prime}\right)\right\}\right]
\end{aligned}
$$

with $\mu^{\prime}(t)=1+P_{\epsilon}(t, t)$ and

$$
\begin{aligned}
& P_{\epsilon}\left(t, t^{\prime}\right)=\epsilon C_{\epsilon}\left(t^{\prime}, 0\right), \\
& \quad+\int_{0}^{t^{\prime}} d s\left[f^{\prime}(t, s) \frac{\partial Q_{\epsilon}\left(t^{\prime}, s\right)}{\partial s}+f^{\prime \prime}(t, s) \frac{\partial Q_{\epsilon}(t, s)}{\partial s} C_{\epsilon}\left(t^{\prime}, s\right)\right],
\end{aligned}
$$

where $f\left(t, t^{\prime}\right) \equiv f\left(C_{\epsilon}\left(t, t^{\prime}\right)\right)$. In the above expression, the temperature $T$ was absorbed to time, so that all quantities in the equations are dimensionless. Integration of Eq. (C2) can be implemented by discretizing the two dimensional plane of the times $\left(t, t^{\prime}\right)$ with $t \geqslant t^{\prime}$ into a cubic lattice of the grid size $\delta$. Note that Eqs. (C2) and (C3) consist of four types of time integrals:

$$
\begin{aligned}
& I^{(1)}\left(t, t^{\prime}\right)=\int_{t^{\prime}}^{t} d s A(t, s) \frac{\partial B\left(s, t^{\prime}\right)}{\partial s}, \\
& I^{(2)}\left(t, t^{\prime}\right)=\int_{t^{\prime}}^{t} d s A(t, s) \frac{\partial B(t, s)}{\partial s} C\left(s, t^{\prime}\right), \\
& I^{(3)}\left(t, t^{\prime}\right)=\int_{0}^{t^{\prime}} d s A(t, s) \frac{\partial B\left(t^{\prime}, s\right)}{\partial s}, \\
& I^{(4)}\left(t, t^{\prime}\right)=\int_{0}^{t^{\prime}} d s A(t, s) \frac{\partial B(t, s)}{\partial s} C\left(t^{\prime}, s\right) .
\end{aligned}
$$

These integrals are evaluated by discretizing the time as $t_{i}=i \delta$ and slicing into pieces as follows. $I^{(1)}\left(t=t_{i}, t^{\prime}=t_{j}\right)$ $\equiv I_{i j}^{(1)}(i>j)$, for example, is written as

$$
\begin{aligned}
I_{i j}^{(1)}= & \int_{t_{m}}^{t_{i}} d s A\left(t_{i}, s\right) \frac{\partial B\left(s, t_{j}\right)}{\partial s}+\int_{t_{j}}^{t_{m}} d s A\left(t_{i}, s\right) \frac{\partial B\left(s, t_{j}\right)}{\partial s} \\
= & A_{i, m} B_{m, j}-A_{i, j} B_{j, j}+\sum_{l=m+1}^{i} \int_{t_{l-1}}^{t_{l}} d s A\left(t_{i}, s\right) \frac{\partial B\left(s, t_{j}\right)}{\partial s} \\
& -\sum_{l=j+1}^{m} \int_{t_{l-1}}^{t_{i}} d s \frac{\partial A\left(t_{i}, s\right)}{\partial s} B\left(s, t_{j}\right)
\end{aligned}
$$

where $m=[(i-j) / 2]$ is the integer closest to but smaller than $(i-j) / 2$. Using an approximation,

$$
\int_{t_{1}}^{t_{2}} d s \frac{\partial A(s)}{\partial s} B(s) \approx\left\{A\left(t_{2}\right)-A\left(t_{1}\right)\right\} \frac{1}{\delta} \int_{t_{1}}^{t_{2}} d s B(s),
$$

which is exact up to $\mathcal{O}\left(\delta^{2}\right),{ }^{49}$ we arrive at

$$
\begin{aligned}
I_{i j}^{(1)}= & A_{i, m} B_{m, j}-A_{i, j} B_{j, j}+\sum_{l=m+1}^{i}\left(B_{l, j}-B_{l-1, j}\right) d A_{i, l}^{(v)} \\
& -\sum_{l=j+1}^{m}\left(A_{i, l}-A_{i, l-1}\right) d B_{l, j}^{(h)}
\end{aligned}
$$

where

$$
\begin{aligned}
& d A_{i j}^{(h)}=\frac{1}{\delta} \int_{t_{i-1}}^{t_{i}} d s A\left(s, t_{j}\right), \\
& d A_{i j}^{(v)}=\frac{1}{\delta} \int_{t_{j-1}}^{t_{j}} d s A\left(t_{i}, s\right)
\end{aligned}
$$

are the integrals over the horizontal and vertical lattice bonds, respectively (we refer to them as bond integrals). Likewise, other integrals can be approximated as follows: 


$$
\begin{aligned}
I_{i j}^{(2)}= & \sum_{l=m+1}^{i} \frac{1}{2} d A_{i, l}^{(v)}\left(B_{i, l}-B_{i, l-1}\right)\left(C_{l, j}+C_{l-1, j}\right)+\sum_{l=j+1}^{m} \frac{1}{2}\left(A_{i, l}\right. \\
& \left.+A_{i, l-1}\right)\left(B_{i, l}-B_{i, l-1}\right) d C_{l, j}^{(h)}, \\
I_{i j}^{(3)}= & A_{i j} B_{j j}-A_{i, 0} B_{j, 0}-\sum_{l=1}^{j}\left(A_{i, l}-A_{i, l-1}\right) d B_{j, l}^{(v)}, \\
I_{i j}^{(4)}= & \sum_{l=1}^{j} \frac{1}{2}\left(A_{i, l}+A_{i, l-1}\right)\left(B_{i, l}-B_{i, l-1}\right) d C_{j, l}^{(v)} .
\end{aligned}
$$

With this discretization, the nonlinear integrodifferential equation, Eq. (C2), can be written in the form of a simultaneous nonlinear equation as

$$
\mathbf{V}_{i}=\mathbf{M}_{i} \cdot \mathbf{F}_{i}\left(\mathbf{V}_{i}\right)+\mathbf{N}_{i},
$$

where $\quad \mathbf{V}_{i}=\left(C_{i 0}, \ldots, C_{i i}, Q_{i 0}, \ldots, Q_{i i}\right) \quad$ and $\quad \mathbf{F}_{i}\left(\mathbf{V}_{i}\right)$ $=\left(f^{\prime}\left(C_{i 0}\right), \ldots, f^{\prime}\left(C_{i i}\right), f^{\prime \prime}\left(C_{i 0}\right), \ldots, f^{\prime \prime}\left(C_{i i}\right)\right) \quad$ are $(2 i+2)$-dimensional vectors. The matrix $\mathbf{M}_{i}$ and the vector $\mathbf{N}_{i}$ are functions of the friction coefficient $\mu^{\prime}$, the vectors at the earlier times $\left(\mathbf{V}_{l}, \mathbf{F}_{l}\right)$ with $l<i$, and a set of bond integrals $\mathbf{W}=\left(d C^{(h)}, d C^{(v)}, d Q^{(h)}, d Q^{(v)}, d f^{\prime(h)}, d f^{\prime(v)}, d f^{\prime \prime(h)}, d f^{\prime \prime(v)}\right)$.

Equation (C10) can be solved self-consistently using the following procedure.

(1) First, prepare the array of exact $\mathbf{V}_{i}, \mathbf{F}_{i}$, and $\mathbf{W}$ for 0 $\leqslant j \leqslant i \leqslant N_{t} / 2$ with a very small time grid $\delta$ such that $N_{t} \delta \ll 1$ by short time expansion of Eq. (C2).

(2) For $i=N_{t} / 2+1$ and for $j$ very close to but smaller than $i$, we import the values of the previous time, expecting the short-time dynamics at $(i-j) \delta \ll 1$ is not affected by the perturbed field or by aging. More specifically, we choose an integer $N_{\text {short }} \ll N_{t} / 2$ and assign the values $C_{i, j}=C_{i-1, j-1}, d C_{i, j}^{(h)}=d C_{i-1, j-1}^{(h)}$ and so forth for $i-N_{\text {short }}$ $\leqslant j \leqslant i$.

(3) For $i=N_{t} / 2+1$ and for $0 \leqslant j<i-N_{\text {short }}$, we solve Eq. (C10) self-consistently by iteration. The iteration is done by choosing the initial array as $\mathbf{V}_{i}=\mathbf{V}_{i-1}$. The bond integrals are calculated using

$$
\begin{aligned}
& d A_{i, j}^{(h)}=\frac{\delta}{12}\left(-A_{i-2, j}+8 A_{i-1, j}+5 A_{i, j}\right), \\
& d A_{i, j}^{(v)}=\frac{\delta}{12}\left(-A_{i, j+2}+8 A_{i, j+1}+5 A_{i, j}\right) .
\end{aligned}
$$

At every iteration of Eq. (C10) for $\mathbf{V}_{i}$, all elements of the bond integrals $d A_{i, j}^{(h, v)}$, and thus $\mathbf{M}$ and $\mathbf{N}$, are updated using Eq. (C11).

(4) Keep procedures (2) and (3) for $N_{t} / 2 \leqslant i \leqslant N_{t}$.

(5) Once all solutions for $0 \leqslant i \leqslant N_{t}$ are obtained, we decimate the number of variables by half in order to save the memory space to explore further for the longer time. We discard half variables and renew all variables by the following rules. For $\mathbf{V}=(C, Q)$,

$$
V_{2 i, 2 j} \rightarrow V_{i, j} \text {. }
$$

For bond integrals,

$$
\begin{aligned}
& \frac{1}{2}\left(d A_{2 i, 2 j}^{(h)}+d A_{2 i-1,2 j}^{(h)}\right) \rightarrow d A_{i, j}^{(h)} \\
& \frac{1}{2}\left(d A_{2 i, 2 j}^{(v)}+d A_{2 i, 2 j-1}^{(v)}\right) \rightarrow d A_{i, j}^{(v)} .
\end{aligned}
$$

Then, the time grid is doubled.

$$
2 \delta \rightarrow \delta \text {. }
$$

(6) Repeat procedures (2)-(5) with the doubled grid size.

We have checked that, in order to obtain a stable result up to the order of $t=10^{16}$ as shown in the present work, we need a a grid number of $N_{t}=1024$ and $N_{\text {short }}=32$, starting from the initial grid size of $\delta=10^{-10}$.

${ }^{1}$ E. Donth, The Glass Transition (Springer, Berlin, 2001).

${ }^{2}$ K. Binder and W. Kob, Glassy Materials and Disordered Solids (World Scientific, Singapore, 2005).

${ }^{3}$ M. D. Ediger, Annu. Rev. Phys. Chem. 51, 99 (2000).

${ }^{4}$ H. Sillescu, J. Non-Cryst. Solids 243, 81 (1999).

${ }^{5}$ R. Richert, J. Phys.: Condens. Matter 14, R703 (2002).

${ }^{6}$ H. C. Andersen, Proc. Natl. Acad. Sci. U.S.A. 102, 6686 (2005).

${ }^{7}$ S. C. Glotzer, J. Non-Cryst. Solids 274, 342 (2000).

${ }^{8}$ B. Doliwa and A. Heuer, Phys. Rev. E 61, 6898 (2000).

${ }^{9}$ R. Yamamoto and A. Onuki, Phys. Rev. Lett. 81, 4915 (1998).

${ }^{10}$ Y. Hiwatari and T. Muranaka, J. Non-Cryst. Solids 235-237, 19 (1998).

${ }^{11}$ S. Franz and G. Parisi, J. Phys.: Condens. Matter 12, 6335 (2000).

${ }^{12}$ S. Franz, C. Donati, G. Parisi, and S. C. Glotzer, Philos. Mag. B 79, 1827 (1999); C. Donati, S. Franz, S. C. Glotzer, and G. Parisi, J. Non-Cryst. Solids 307, 215 (2002).

${ }^{13}$ J. P. Garrahan and D. Chandler, Phys. Rev. Lett. 89, 035704 (2002).

${ }^{14}$ C. Toninelli, M. Wyart, G. Biroli, L. Berthier, and J.-P. Bouchaud, Phys. Rev. E 71, 041505 (2005).

${ }^{15}$ L. Berthier, Phys. Rev. E 69, 020201 (2004).

${ }^{16}$ L. Berthier, G. Biroli, J.-P. Bouchaud, W. Kob, K. Miyazaki, and D. R. Reichman, J. Chem. Phys. 126, 184503 (2007), preceding paper.

${ }^{17}$ P. Mayer, H. Bissig, L. Berthier, L. Cipelletti, J. P. Garrahan, P. Sollich, and V. Trappe, Phys. Rev. Lett. 93, 115701 (2004).

${ }^{18}$ O. Dauchot, G. Marty, and G. Biroli, Phys. Rev. Lett. 95, 265701 (2005).

${ }^{19}$ L. Berthier, G. Biroli, J.-P. Bouchaud, L. Cipelletti, D. El Masri, D. L'Hôte, F. Ladieu, and M. Pierno, Science 310, 1797 (2005).

${ }^{20}$ A. Duri and L. Cipelletti, Europhys. Lett. 76, 972 (2006).

${ }^{21}$ J. P. Hansen and I. R. McDonald, Theory of Simple Liquids (Elsevier, Amsterdam, 1986).

${ }^{22}$ J. L. Lebowitz, J. K. Percus, and L. Verlet, Phys. Rev. 153, 250 (1967).

${ }^{23}$ T. Gleim, W. Kob, and K. Binder, Phys. Rev. Lett. 81, 4404 (1998).

${ }^{24}$ G. Szamel and E. Flenner, Europhys. Lett. 67, 779 (2004).

${ }^{25}$ L. Berthier and W. Kob, J. Phys.: Condens. Matter (to be published); cond-mat/0610253.

${ }^{26}$ J.-P. Bouchaud, L. F. Cugliandolo, J. Kurchan, and M. Mézard, Physica A 226, 243 (1996).

${ }^{27}$ W. Götze, J. Phys.: Condens. Matter 11, A1 (1999); W. Götze and L. Sjögren, Rep. Prog. Phys. 55, 241 (1992).

${ }^{28}$ F. Ritort and P. Sollich, Adv. Phys. 52, 219 (2003).

${ }^{29}$ G. Szamel and H. Löwen, Phys. Rev. A 44, 8215 (1991).

${ }^{30}$ A. Montanari and G. Semerjian, J. Stat. Phys. 125, 23 (2006).

${ }^{31}$ G. Biroli, J.-P. Bouchaud, K. Miyazaki, and D. R. Reichman, Phys. Rev. Lett. 97, 195701 (2006).

${ }^{32}$ T. R. Kirkpatrick and D. Thirumalai, Phys. Rev. A 37, 4439 (1988).

${ }^{33}$ T. R. Kirkpatrick, D. Thirumalai, and P. G. Wolynes, Phys. Rev. A 40, 1045 (1989).

${ }^{34}$ G. Biroli and J. P. Bouchaud, Europhys. Lett. 67, 21 (2004).

${ }^{35}$ K. Kawasaki, Physica A 208, 35 (1994).

${ }^{36}$ D. S. Dean, J. Phys. A 29, L613 (1996).

${ }^{37}$ A. Andreanov, G. Biroli, and A. Lefèvre, J. Stat. Mech.: Theory Exp. 2006, P07008.

${ }^{38}$ S. P. Das, Rev. Mod. Phys. 76, 785 (2004).

${ }^{39}$ K. Miyazaki and D. R. Reichman, J. Phys. A 38, L343 (2005).

${ }^{40}$ J. Zinn-Justin, Quantum Field Theory and Critical Phenomena (Oxford University Press, Oxford, 2002).

${ }^{41}$ D. R. Reichman and P. Charbonneau, J. Stat. Mech.: Theory Exp. 2005, P05013. 
${ }^{42}$ C. De Dominicis and P. C. Martin, J. Math. Phys. 5, 14 (1964); 5, 31 (1964).

${ }^{43}$ J.-P. Blaizot and G. Ripka, Quantum Theory of Finite Systems (Editions Phenix, Kiev, 1998)

${ }^{44}$ G. Biroli and J.-P. Bouchaud, cond-mat/0609705.

${ }^{45}$ C. Bennemann, C. Donati, J. Baschnagel, and S. C. Glotzer, Nature (London) 399, 246 (1999).

${ }^{46}$ N. Lačević, F. W. Starr, T. B. Schrøder, and S. C. Glotzer, J. Chem. Phys. 119, 7372 (2003).

${ }^{47}$ S. Whitelam, L. Berthier, and J. P. Garrahan, Phys. Rev. Lett. 92, 185705 (2004).

${ }^{48}$ D. Chandler, J. P. Garrahan, R. L. Jack, L. Maibaum, and A. C. Pan, Phys. Rev. E 74, 051501 (2006)

${ }^{49}$ M. Fuchs, W. Götze, I. Hofacker, and A. Latz, J. Phys.: Condens. Matter 3, 5047 (1991)

${ }^{50}$ J. Jäckle and S. Eisinger, Z. Phys. B: Condens. Matter 84, 115 (1991).

${ }^{51}$ G. H. Fredrickson and H. C. Andersen, Phys. Rev. Lett. 53, 1244 (1984); J. Chem. Phys. 83, 5822 (1985).

${ }^{52}$ S. Butler and P. Harrowell, J. Chem. Phys. 95, 4454 (1991); 95, 4466 (1991); P. Harrowell, Phys. Rev. E 48, 4359 (1993); M. Foley and P. Harrowell, J. Chem. Phys. 98, 5069 (1993).

${ }^{53}$ M. Merolle, J. P. Garrahan, and D. Chandler, Proc. Natl. Acad. Sci. U.S.A. 102, 10837 (2005).

${ }^{54}$ L. Berthier and J. P. Garrahan, J. Chem. Phys. 119, 4367 (2003); Phys. Rev. E 68, 041201 (2003).

${ }^{55}$ C. Toninelli, G. Biroli, and D. S. Fisher, Phys. Rev. Lett. 92, 185504 (2004); J. Stat. Phys. 120, 167 (2005).

${ }^{56}$ M. Sellitto, G. Biroli, and C. Toninelli, Europhys. Lett. 69, 496 (2005).

${ }^{57}$ W. Kob and H. C. Andersen, Phys. Rev. E 48, 4364 (1993).

${ }^{58}$ S. Whitelam, L. Berthier, and J. P. Garrahan, Phys. Rev. E 71, 026128 (2005).

${ }^{59}$ R. L. Jack, P. Mayer, and P. Sollich, J. Stat. Mech.: Theory Exp. 2006, P03006.

${ }^{60}$ N. Cancrini, F. Martinelli, C. Roberto, and C. Toninelli, J. Stat. Mech.: Theory Exp. 2007, L03001.

${ }^{61}$ S. N. Taraskin and S. R. Elliott, Europhys. Lett. 39, 37 (1997); M. Benoit, S. Ispas, P. Jund, and R. Jullien, Eur. Phys. J. B 13, 631 (2000); J. Horbach and W. Kob, Phys. Rev. B 60, 3169 (1999); Phys. Rev. E 64, 041503 (2001)

${ }^{62}$ U. Geyer, W. L. Johnson, S. Schneider, Y. Qui, T. A. Tombrello, and M.-P. Macht, Appl. Phys. Lett. 69, 2492 (1996).

${ }^{63}$ L. Berthier and J. P. Garrahan, J. Phys. Chem. B 109, 3578 (2005).

${ }^{64}$ A. Buhot and J. P. Garrahan, Phys. Rev. E 64, 21505 (2001).

${ }^{65}$ P. Sollich and M. R. Evans, Phys. Rev. E 68, 031504 (2003).

${ }^{66}$ C. Toninelli, G. Biroli, and D. S. Fisher, Phys. Rev. Lett. 96, 035702 (2006)

${ }^{67}$ L. Berthier, Phys. Rev. Lett. 91, 055701 (2003).

${ }^{68}$ P. De Gregorio, A. Lawlor, P. Bradley, and K. A. Dawson, Phys. Rev. Lett. 93, 025501 (2004).

${ }^{69}$ L. Berthier, G. Biroli, and C. Toninelli (unpublished).
${ }^{70}$ J. P. Garrahan and D. Chandler, Proc. Natl. Acad. Sci. U.S.A. 100, 9710 (2003).

${ }^{71}$ G. Szamel and E. Flenner, Phys. Rev. E 74, 021507 (2006).

${ }^{72}$ G. Biroli, J.-P. Bouchaud, and G. Tarjus, J. Chem. Phys. 123, 044510 (2005).

${ }^{73}$ D. Chandler and J.-P. Garrahan, J. Chem. Phys. 123, 044511 (2005).

${ }^{74}$ W. Kob and H. C. Andersen, Phys. Rev. Lett. 73, 1376 (1994); Phys. Rev. E 53, 4134 (1995); 52, 4626 (1995).

${ }^{75}$ B. W. H. van Beest, G. J. Kramer, and R. A. van Santen, Phys. Rev. Lett. 64, 1955 (1990).

${ }^{76}$ M. P. Allen and D. J. Tildesley, Computer Simulation of Liquids (Oxford University Press, Oxford, 1987).

${ }^{77}$ M. Nauroth and W. Kob, Phys. Rev. E 55, 657 (1997).

${ }^{78}$ C. Alba-Simioneso, L. Berthier, G. Biroli, J.-P. Bouchaud, C. DalleFerrier, D. L'Hôte, F. Ladieu, G. Tarjus, and C. Thibierge (unpublished).

${ }^{79}$ X. Y. Xia and P. G. Wolynes, Proc. Natl. Acad. Sci. U.S.A. 97, 2990 (2000).

${ }^{80}$ P. Viot, G. Tarjus, and D. Kivelson, J. Chem. Phys. 112, 10368 (2000).

${ }^{81}$ J.-P. Bouchaud and G. Biroli, J. Chem. Phys. 121, 7347 (2004).

${ }^{82}$ U. Tracht, M. Wilhelm, A. Heuer, H. Feng, K. Schmidt-Rohr, and H. W. Spiess, Phys. Rev. Lett. 81, 2727 (1998).

${ }^{83}$ S. A. Reinsberg, X. H. Qiu, M. Wilhelm, H. W. Spiess, and M. D. Ediger, J. Chem. Phys. 114, 7299 (2001).

${ }^{84}$ X. H. Qiu and M. D. Ediger, J. Phys. Chem. B 107, 459 (2003).

${ }^{85}$ J. D. Stevenson, J. Schmalian, and P. G. Wolynes, Nat. Phys. 2, 268 (2006).

${ }^{86}$ P. Chandra, M. V. Feigelman, and L. B. Ioffe, Phys. Rev. Lett. 76, 4805 (1996).

${ }^{87}$ A. V. Lopatin and L. B. Ioffe, Phys. Rev. B 66, 174202 (2002).

${ }^{88}$ K.-K. Loh, K. Kawasaki, A. R. Bishop, T. Lookman, A. Saxena, Z. Nussinov, and J. Schmalian, Phys. Rev. E 69, 010501(R) (2004).

${ }^{89}$ S. Franz and F. L. Toninelli, Phys. Rev. Lett. 92, 030602 (2004); J. Phys. A 37, 7433 (2004).

${ }^{90}$ B. Kim and A. Latz, Europhys. Lett. 53, 660 (2001).

${ }^{91}$ Note that $\left\{C-B^{\dagger} A^{-1} B\right\}$ is the propagator of the theory but it coincides with $G$, the solution of the self-consistent equation, only if all diagrams are retained. Within the MCT approximation they are quantitatively different but qualitatively similar. The self-energy $B^{\dagger} A^{-1} B$ contains ladder diagrams but they are harmless because the times on the same side of the ladders coincide since they are attached to the same vertex. Therefore, they are related to the dynamical fluctuations at equal time which are not critical.

${ }^{92}$ Note that, within MCT, $T_{\chi T} / \sqrt{c_{V}}$ and $\chi_{T}$ have the same scaling when approaching the critical point. In real materials, the MCT transition (if any) is avoided and temperature-dependent prefactors might slightly change the observed scaling behavior. Here we have chosen to focus on $T_{\chi T} / \sqrt{c_{V}}$ because it corresponds to a properly normalized correlation function. 